\title{
A Model to Predict Liquid Bridge Formation Between Wet Particles Based on Direct Numerical Simulations
}

\author{
Mingqiu Wu and Stefan Radl \\ Institute of Process and Particle Engineering, Graz University of Technology, Inffeldgasse 13/III, 8010 Graz, \\ Austria \\ Johannes Khinast \\ Institute of Process and Particle Engineering and Research Center Pharmaceutical Engineering GmbH, Graz \\ University of Technology, Inffeldgasse 13/III, 8010Graz, Austria
}

DOI 10.1002/aic.15184

Published online in Wiley Online Library (wileyonlinelibrary.com)

\begin{abstract}
We study dynamic liquid bridge formation, which is relevant for wet granular flows involving highly viscous liquids and short collisions. Specifically, the drainage process of liquid adhering to two identical, non-porous wet particles with difference initial film heights is simulated using Direct Numerical Simulations (DNS). We extract the position of the interface, and define the liquid bridge and its volume by detecting a characteristic neck position. This allows us building a dynamic model for predicting bridge volume, and the liquid remaining on the particle surface. Our model is based on two dimensionless mobility parameters, as well as a dimensionless time scale to describe the filling process. In the present work model parameters were calibrated with DNS data. We find that the proposed model structure is sufficient to collapse all our simulation data, indicating that our model is general enough to describe liquid bridge formation between equally sized particles. (C) 2016 American Institute of Chemical Engineers AIChE J, 00: 000-000, 2016

Keywords: granular flows, wet particles, liquid bridge, liquid transport, direct numerical simulation, volume of fluid method
\end{abstract}

\section{Introduction}

Flow of highly saturated wet granular matter is encountered in a wide range of engineering applications, particularly in the energy sector, or the pharmaceutics and food industry. ${ }^{1}$ Due to viscous effects, evaporation or condensation, capillary forces and inhomogeneous liquid distribution in wet granular flows, the liquid transport is difficult to describe and complex flow behaviour is generally observed. ${ }^{2}$ Specifically, liquid bridges between particles may lead to particle agglomeration ${ }^{3}$ which is either wanted (in the case of wet granulation), or unwanted (e.g., in wet fluidized beds used for coking). Clearly, a better understanding of the formation of liquid bridges will aid in controlling these processes. Previous studies on liquid bridges between particles mainly focused on static bridges, ${ }^{4}$ bridge deformation during stretching and rupture, ${ }^{2,5-7}$ or the energy dissipated on rupture, ${ }^{2,8,9}$ however, few theoretical and experimental studies provided a detailed understanding of the initial bridge formation process, and the accompanying liquid transfer rate from the particle surface into the bridge. Experimental results, and the resulting empirical models, have been summarized by Herminghaus ${ }^{10}$, mainly focusing on the effect of roughness, as well as evaporation and re-condensation. Unfortunately, these models cannot be applied to engineering problems, since they (1) focus on the long-term behaviour of

Correspondence concerning this article should be addressed to S. Radl at radl@ tugraz.at.

(C) 2016 American Institute of Chemical Engineers the bridge and (2) do not provide a closure for the model parameters.

Studies of liquid bridges between two identical particles were initiated in $1920 \mathrm{~s}^{11,12}$ and were later extended to cover pendular bridges between unequal-sized particles, or a particle and a wall. ${ }^{4,13}$ Studies of moving particles and the associated liquid bridge formation were carried out, starting with the work of Pitois et al. ${ }^{14}$, which found that the particle relative velocity significantly influences the liquid bridge force. Rossetti and Simons ${ }^{9}$ introduced an important improvement, that is, a novel micro force balance device which is capable of observing the liquid bridge between particles and measuring the force exerted by liquid bride. Darabi et al. ${ }^{2}$, presented a new coalescence model for binary collision between two identical wet particles, considering capillary and viscous forces exerted by (instantaneously formed) pendular bridges. Despite a variety of researchers ${ }^{7,15-22}$ that has broadened our understanding of liquid bridges, a study describing a detailed model on the time evolution of a single (pendular) liquid bridge during its formation phase is still missing. This is due to the lack of our understanding how quickly liquid is transported into a liquid bridge, and how much of the liquid (initially present on the particles) is able to flow into the bridge. One reason for this lack of understanding is that experimental investigations to quantify the liquid present in the bridge are tedious, and that simulations require an enormous spatial and temporal resolution to picture the filling process.

Wet particle collisions, or collisions in a viscous fluid, have been studied by a variety of researchers. For example, Davis

\section{0}


et al. $^{23}$, experimentally studied particle collisions with wet smooth surfaces, and they demonstrate that the lubrication forces play an important role on the particle rebound. Donahue et al. $^{24}$, further studied the collisions between liquid-coated spheres using a three-body Newton's Cradle, and they revealed that fluid lubrication resistance on rebound plays a key role in the dynamics of the collision. Li et al. ${ }^{25}$, calibrated a model with experimental results of wet particle collisions. Gondret et al. ${ }^{26}$, focused on the bouncing motion of spherical particles in a viscous fluid, and they quantified the wet restitution coefficient by varying the density and the elastic properties of the solid sphere, as well as liquid film viscosity. Gollwitzer et al. ${ }^{27}$, revealed that the dimensionless film thickness is a crucial parameter that affects the restitution coefficient. A rough estimate of the liquid bridge volume, i.e., $V_{b} \approx d_{p}{ }^{3} / 16$, was used in their work to determine the rupture energy. Sutkar et al. ${ }^{28}$ provided a new approach for the estimation of wet restitution coefficients between a particle and a wet surface based on a dimensional and energy budget analysis. However, their data is only in fair agreement with the proposed model, and they have not provided a model for the prediction of the liquid bridge volume. In summary, a large number of researchers have focused on particle collision dynamics without a detailed analysis of liquid bridge formation. ${ }^{25,29,30}$

In this article we focus on applying simulations to elucidate the complex flow processes associated with bridge formation. In general, three categories of simulations methods that aim at modeling liquid bridges can be distinguished: (1) solving the Young-Laplace equation (YLE), (2) solving an approximated version of the YLE based on geometrical simplifications, and (3) a full numerical solution of the Navier-Stokes equation of the gas-liquid systems.

The first approach yields analytical solutions only for certain geometrical configurations, ${ }^{4}$ and hence, one has to employ a numerical integration for a general geometrical configuration. ${ }^{31-33}$ For example, Lian et al. ${ }^{32}$, provided a simple numerical scheme for solving the YLE and revealed that the rupture distance of equally-sized particles can be written as the cube root of the liquid bridge volume for small contact angles. They also obtained a bridge force model based on a simple approximation method, which was independent of the contact angle. Similarly, Mikami et al. ${ }^{34}$, and Willett et al. ${ }^{35}$, proposed a simple capillary force model by fitting the numerical solution of the YLE. However, as shown in Pepin et al. ${ }^{8}$, the drawbacks when solving the YLE to study liquid bridges are that (1) a fixed contact angles is required and (2) that the effect of liquid flow into (or out of) the bridge cannot be predicted.

The second approach is to obtain a simple approximation of the liquid bridge shape, typically by assuming a toroidal ${ }^{13,36,37}$ or parabolic shape (see, e.g., Pepin et al. ${ }^{8}$ ). The benefits of using simplified approximation models are obvious: there is no need to solve the full YLE and an analytical solution can be obtained that can be readily implemented into particles simulation codes. However, approximate solutions become increasingly inaccurate for increasing particle separation ${ }^{38}$ and they suffer the same drawbacks as the solution of the YLE.

The third approach is the numerical solution of the NavierStokes equations describing (1) the flow of the liquid, or (2) the liquid and the surrounding gas. In the past two decades this approach has been used with increasing frequency. Early research was based on a simplified version of the NavierStokes equation, that is, modified boundary conditions were used at the interface (see, e.g., Eggers and Dupont ${ }^{39}$ as well as 141 Papageorgiou $\left.^{40}\right)$. Later, Zhang et al. ${ }^{41}$, investigated the 142 stretching of a liquid bridge between two circular disks by 143 using a similar method. A fair amount of work to simplify the 144 Navier-Stokes equations for the situation of liquid bridges 145 between two circular disks (but not actual spherical particles) 146 has been performed. Hence, these previous work is only able 147 to provide qualitative information, and has little value for 148 practical application. Only very recently, direct numerical 149 simulations (DNS) of the Navier-Stokes equation have been 150 attempted to simulate liquid bridge formation in an axisym- 151 metric setup. ${ }^{42-45}$ These methods provide a full description of 152 the liquid bridge dynamics, and hence are a promising 153 approach for studying the bridge formation process.

\section{Objectives}

Only few theoretical and experimental studies in the litera- 156 ture were concerned with the bridge formation process and the 157 accompanying liquid transfer rate into the bridge. While mod- 158 els for liquid transfer on bridge rupture exist Lian et al. ${ }^{32}$, as 159 well as Shi and McCarthy ${ }^{46}$, these models still require addi- 160 tional assumptions for the liquid volume present in the bridge. 161 With this in mind, we have started investigations to establish a 162 detailed model that is able to predict liquid transport and the 163 distribution of liquid between two spheres in our recent 164 work. ${ }^{43}$ In the present work, we systematically study the liquid 165 bridge and drainage process of liquid adhering to two identical 166 wet particles. We use a DNS based on the Volume of Fluid 167 (VoF) method, that is, we simulate both the motion of the liq- 168 uid and the surrounding gas. By reconstructing the interface 169 between these two fluid phases, we extract the interface posi- 170 tion to identify the bridge shape and size. Specifically, the liq- 171 uid bridge is defined by detecting the neck positions of the 172 liquid film on each particle surface (for details see the "Liquid 173 Bridge Volume calculation" section). We then use a direct 174 integration method (DIM) to calculate the liquid bridge vol- 175 ume based on the interface position at each instant in time. 176 Our ultimate goal is then building a dynamic model for the liq- 177 uid bridge volume during the filling process based on these 178 DNS data. Therefore, we fit our DNS data to a postulated liq- 179 uid bridge filling model, which is an extension of the ideas of 180 Mohan et al. ${ }^{47}$, but still allows for an analytical solution to 181 predict the bridge volume. Specifically, our postulated model 182 assumes that the filling rate is not affected by the particles' rel- 183 ative motion, and that the filling rate is linear in the difference 184 between the liquid present on a particle and the bridge volume. 185 Such a model requires the specification of only three dimen- 186 sionless parameters, as we will show in the following. While 187 our work is currently limited to smooth particles of identical 188 size, the model proposed by us can be easily re-calibrated to 189 account for, that is, particle roughness, or particle size differ- 190 ences once data for the amount of liquid in the bridge is available for these situations.

In the following, we first describe the methodology used to 193 establish the liquid bridge model, including (1) the initial 194 bridge and boundary conditions that have been used in our 195 simulations, (2) the interface feature extraction procedure, as 196 well as (3) the postulated model itself. Subsequently, we intro- 197 duce a geometrical bridge volume, which is used to normalize 198 the bridge volume measured from our detailed simulations. 199 Then we present results, starting with the calibration of the 200 sub-models for the initial bridge volume and the subsequent 201 viscous filling stage, and we are the first to provide a dynamic 202 


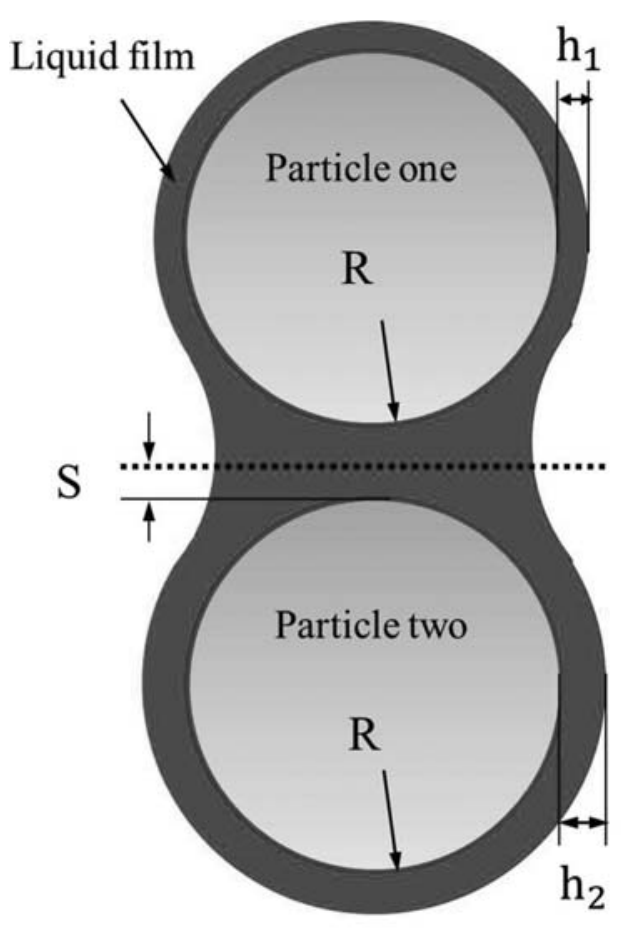

(a)

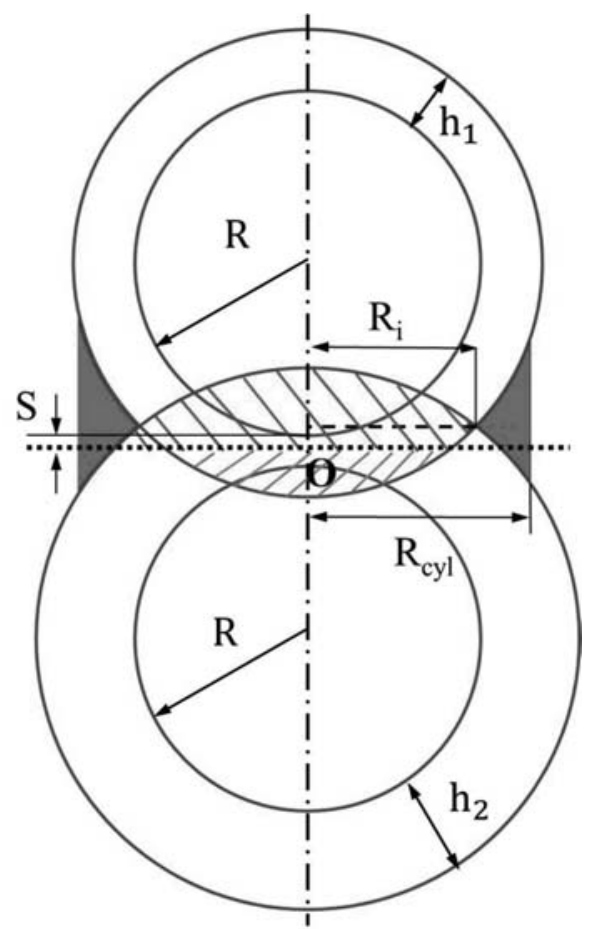

(b)

Figure 1. Sketch of the simulation setup, as well as the initial bridge shape.

[Color figure can be viewed in the online issue, which is available at wileyonlinelibrary.com.]

model for predicting liquid bridge formation. Also, the effect of grid refinement, as well as that of the Reynolds number, are carefully analyzed as well. Finally, we discuss our findings and provide conclusions that should guide the application and future extension of our model.

\section{Methodology \\ Initial and boundary conditions}

We consider two identical smooth spheres, which are fixed in space, that is, the spheres' relative velocity is zero. In this work, we consider the general situation of an asymmetric liquid bridge, that is, the thickness of the films initially present on the particle surfaces are different. In what follows, we define that particle 1 always has less liquid compared to particle 2, as shown in Figure 1 (panel a). $R$ is the sphere radius for two particles, and $h_{1}$ and $h_{2}$ are the initial film heights of particle 1 and particle 2, respectively. $S$ is defined as the half separation distance between the particle surfaces.

The initial shape of the liquid bridge has been set according to the initial film height and the particle separation. Because we do not simulate the approach of the spheres, and hence cannot predict the deformation of the liquid films on the particle prior to coalescence, we must assume the initial bridge shape right after the films have coalesced. Specifically, we assume that the liquid in the overlapping region of the liquid films (i.e., red and green shaded regions in Figure 1b) is instantaneously displaced laterally, and flows into a ringshaped region. The latter is illustrated by the red solid area in Figure $1 \mathrm{~b}$, that is, the bridge has been considered to be cylindrical at time zero. Geometrical considerations, discussed in greater detail in "Geometrical Bridge Volume" section, can now be used to predict the size of the ring-shaped region. These considerations, as well as the assumption of zero initial velocity and uniform pressure distribution, have been used to 235 initialize all our simulations.

Most important, in our simulations there is no gravity, or 237 other force acting on the system. The physical reason why liq- 238 uid in the films on the particle surfaces flows into the liquid 239 bridge is as follows: the pressure in the film (adhering to the 240 particle surface, and far away from the bridge) can be esti- 241 mated as $p_{s} \approx 2 \sigma / R$, while the pressure in the liquid bridge 242 region can be approximated as $p_{V_{b}} \approx-\sigma / R_{\text {curve. }}$. Here $R_{\text {curve }}$ is 243 the radius of curvature of the liquid bridge surface. Thus, the 244 pressure in the liquid bridge region is always negative or zero, 245 while that in the film is always positive. Hence, a pressure dif- 246 ference between the particle surface and the liquid bridge 247 region exists, driving the liquid into the bridge. This liquid 248 flow will not stop until the pressure difference reaches zero, or 249 the liquid film on the particle surface ruptures (for details 250 about film rupture see section "Film Rupture and Grid 251 Refinement").

\section{VoF simulation approach}

A VoF method, ${ }^{48}$ which is available as "interFoam" solver 254 in the open-source software package OpenFOAM ${ }^{\circledR}$, has been 255 employed in our simulation. The interFoam solver has been 256 verified extensively by Deshpande et al. ${ }^{48}$, and we have also 257 made several tests, for example, calculating the pressure distri- 258 bution in a liquid film coating a single sphere. For a typical 259 grid resolution of $\Delta h=\Delta x / h_{l}=0.10$ (here $\Delta x$ is the grid spac- 260 ing, and $h$ is the film height), these test show that the pressure 261 can be predicted within an acceptable error tolerance of ca. 262 $-4.6 \%$. In addition, we have tested the grid dependency of 263 our results, and found that $\Delta h=0.12$ gives acceptable results 264 for most situations of interest (see section "Film Rupture and 265 Grid Refinement" for more details). . 
Another critical point when it comes to two-phase flows is the prediction of the dynamic contact angle, since this is not a constant, but is influenced by the speed of the three-phase contact line. Unfortunately, the current implementation of dynamic contact angles in the "interFoam" solver has not been verified, and hence we are unable to accurately simulate the motion of three-phase contact lines. However, we have spared out this detailed by simply assuming that the spheres are completely coated, and hence there is (initially) no three-phase contact line in our simulations. This allows us to apply the "interFoam" solver for our studies of two coated particles without additional modifications. It must be mentioned here that for long times we observe a rupture of the liquid film present on the spheres. In such a situation a three-phase contact line forms, and hence, our solver will deliver inaccurate (but still physical) predictions of the liquid bridge shape after rupture. We accept this inaccuracy for the time being, and simply have not considered data collected after film rupturing events in our analysis.

The transport equation for a color function, representing the volume fraction of the liquid phase, is solved together with the continuity and momentum equations:

$$
\begin{gathered}
\frac{\partial(\rho \boldsymbol{U})}{\partial t}+\nabla \cdot(\rho \boldsymbol{U} \boldsymbol{U})=-\nabla p+\mu\left[\nabla \boldsymbol{U}+(\nabla \boldsymbol{U})^{T}\right]+\boldsymbol{f}_{b} \\
\nabla \cdot \boldsymbol{U}=0 \\
\frac{\partial \alpha}{\partial t}+\nabla \cdot(\boldsymbol{U} \alpha)=0
\end{gathered}
$$

where $\boldsymbol{U}$ is the velocity field shared by the two fluids (i.e., the liquid on the spheres and the surrounding gas) throughout the flow domain, and $\alpha$ is the phase fraction indicator. $\rho$ is density, $p$ is pressure, and $f_{b}$ are body forces, which include (1) surface tension effects at the interface, and (2) gravity. However, effects due to gravity have been neglected in our simulation, because viscous and capillary effects are dominant in situations involving relevant particles (i.e., in situations in which particles have a diameter that is smaller than the capillary length). The phase function $\alpha$ can proceed within the range $0<\alpha<1$, with alpha being zero (or unity) in regions occupied by the gas (or the liquid), respectively.

The physical properties are computed as averages based on the distribution of the liquid volume fraction $\alpha$. Specifically, we use:

$$
\begin{aligned}
& \rho=\rho_{l} \alpha+\rho_{g}(1-\alpha) \\
& \mu=\mu_{l} \alpha+\mu_{g}(1-\alpha)
\end{aligned}
$$

where $\rho_{l}$ (or $\mu_{l}$ ) and $\rho_{g}$ (or $\mu_{g}$ ) are the density (or the dynamic viscosity) of the liquid and gas, respectively.

Relevant dimensionless quantities describing the problem can be readily identified: the dimensionless initial film heights, the dimensionless separation distance, the bridge volume and the amount of liquid on particle surface normalized by a reference volume (i.e., the particle radius cubed $R^{3}$ ), the density and viscosity ratio between the liquid and the ambient gas, the pressure scaled with a typical capillary pressure (i.e., surface tension over the particle radius) and the velocity scaled with a typical capillary speed (i.e., the ratio of surface tension and viscosity of the liquid). The relevant time scale can be based on a corresponding viscous time scale, which is chosen to be the ratio of the particle radius and the capillary speed. Finally, we may want to consider the effect of the fluid's inertia on the filling process, and hence a Reynolds number can be defined based on the capillary speed, the particle radius, and the fluid 318 viscosity. Note, that alternatively an Ohnesorge number can 319 be defined, with is simply the inverse of the square root of the 320 Reynolds number as summarized below:

- $h_{1}^{+}=h_{1} / R, h_{2}^{+}=h_{2} / R, h_{0}^{+}=\left(h_{1}^{+}+h_{2}^{+}\right) / 2, S^{+}=S / R$;

- $L_{p 1}^{+}=L_{p 1} / R^{3}, L_{p 2}^{+}=L_{p 2} / R^{3}, V_{b}^{+}=V_{b} / R^{3}$;

- $\rho_{\text {ratio }}=\rho_{l} / \rho_{g}, \mu_{\text {ratio }}=\mu_{l} / \mu_{g}$;

- $u_{\text {ref }}=\sigma / \mu_{l}, p_{\text {ref }}=\sigma / R, t_{\text {ref }}=R \mu_{l} / \sigma$;

- $t^{+}=t / t_{\mathrm{ref}}$

- $\operatorname{Re}=\sigma R \rho_{l} / \mu_{l}^{2}, O h=\mu_{l} / \sqrt{\rho_{l} \sigma R}=1 / \sqrt{\operatorname{Re}}, C a=\mu_{l}|U| / \sigma$.

A typical result of our DNS is shown in Figure 2, in which 328 F2 we illustrate the dimensionless flow velocity for various 329 dimensionless times. Most important, this figure illustrates 330 that the dimensionless velocity strongly decrease with time, 331 and it can be expected that the rate with which the bridge is 332 filled decreases with increasing time.

All relevant simulation parameters and numerical scheme are defined in Table 1.

\section{Liquid bridge volume calculation}

The gas-liquid interface can be easily determined from the DNS data by analyzing the distribution of the phase fraction. Hence, we have taken a simple, yet effective sampling method to detect the gas-liquid of the film and the bridge formed between the particles.

As can be seen in Figure 3, the sampling procedure takes place between $\mathrm{O}_{1}$ and $\mathrm{O}_{2}$ with an interval of $\delta x$ and a large enough sample distance in the $y$-direction. By applying the sampling interval from $\mathrm{O}_{1}$ and $\mathrm{O}_{2}$, we obtain a list of data for the phase value along each sampling line, and consequently the interface position can be determined at $\alpha=0.5$.

Next, we need to define which portion of the fluid in the system is considered to be in the liquid bridge. As can be seen from Figures 2 and 3 (panel b), there exists a minimal liquid film thickness on each of the two spheres. Thus, if one would analyze the thickness profile on each particle, one can observe a certain angular position where the film is thinnest. We have used this local minimum to define the extent of the liquid bridge. Specifically, we denote these positions of the minima as the "neck" positions, which separate the bridge from the film adhering to the particle surface. These neck positions are the basis for the subsequent bridge volume calculation.

After the interface positions and neck position have been 359 determined, we can calculate the liquid bridge volume by 360 using a DIM. Specifically, we use slices with thickness $\delta x$ (see 361 Figure 3, panel b), as well as the known neck positions, to 362 determine the bridge volume by numerical integration using 363 the trapezoidal rule.

\section{Proposed model for liquid bridge filling}

Our DNS indicate that the mechanism of liquid bridge for- 366 mation consists of the following steps: after the coated par- 367 ticles get close to each other (caused by the relative motion in 368 a real-world granular flow), the films coalesce, a liquid bridge 369 is then formed between the particles, and finally liquid drains 370 into the bridge. Thus, it is reasonable to assume that there is 371 (1) a very fast initial formation processes (immediately after 372 coalescence; since we cannot resolve this process, we will 373 simply assume a certain initial bridge volume), and (2) and a 374 comparably slow filling process. Consequently, we differenti- 375 ate between two stages of the filling process of liquid bridge: 376 (I) a capillary-force driven initial stage (fast filling), and (II) a 377 viscous filling stage (slower filling). Although these two stages 378 


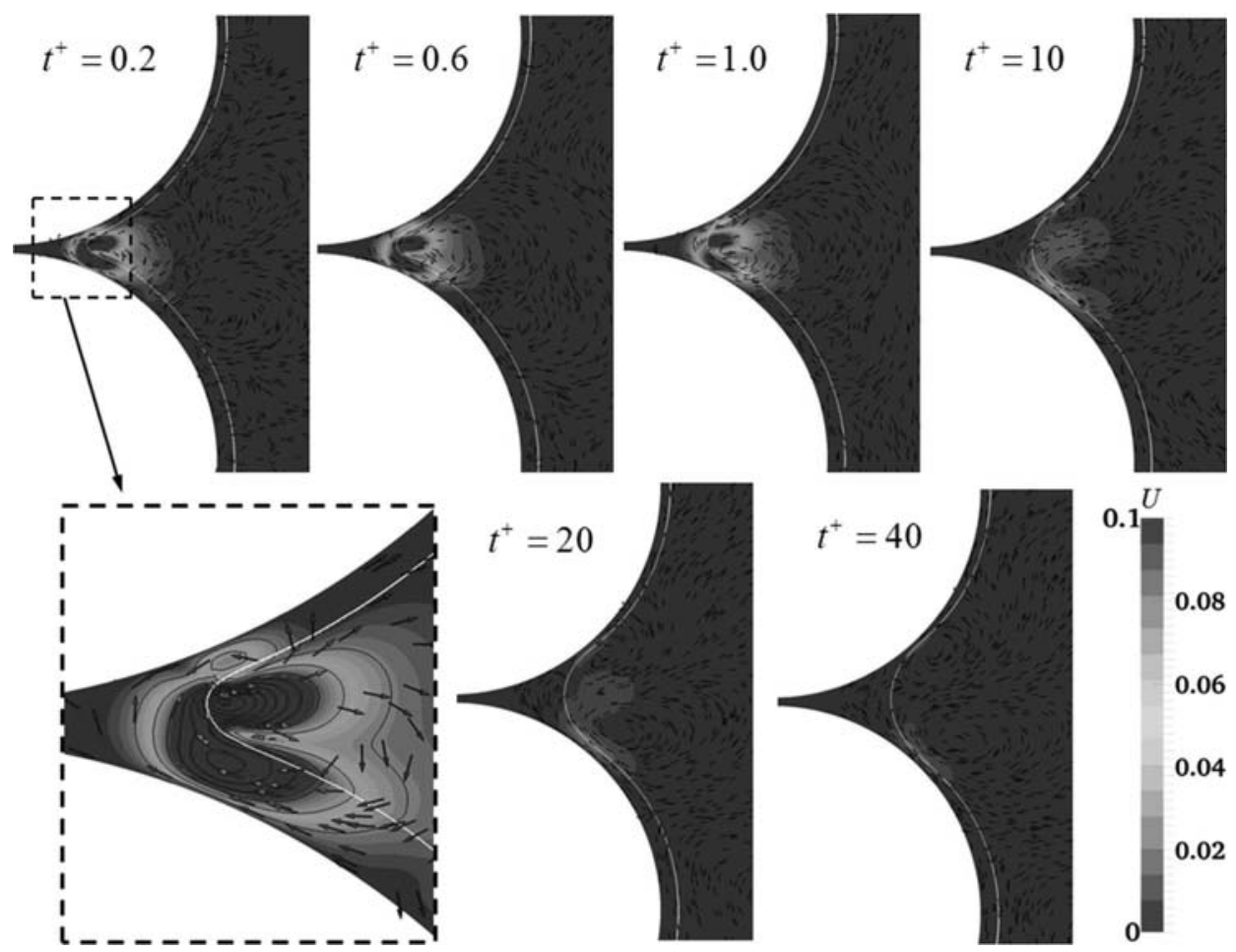

Figure 2. Typical velocity field for fluids flow over two fully coated particles using DNS.

[Color figure can be viewed in the online issue, which is available at wileyonlinelibrary.com.]

will overlap in physical reality, we define stage I to end after a (viscous) reference time scale of $t_{\text {ref }}$, that is, a dimensionless time of $t^{+}=1$. As shown next, we employ two different submodels to predict the liquid bridge volume in these two stages. By employing an overall mass balance it is then straight forward to predict the liquid residing on the contacting particles.

For the initial stage we simply aim on correlating the bridge volume after $t^{+}=1$ with the most important process parameters. Specifically, we simply choose the key geometrical parameters, which are (1) the initial film height $h_{0}$, as well as (2) the half separation distance $S$.

The next section of the article details on a postulated model to predict the time evolution of liquid bridge volume in stage II. Specifically, we use a phenomenological closure for the flow rate between the film and bridge compartment. We assume the flow rate to be proportional to the difference of the mobile fraction of the liquid on the particle and half of the bridge volume. To compute the mobile fraction of the liquid present on the particle, we use a single parameter which is called the mobility parameter $\phi_{m, i}$. This parameter is simply the ratio of the mobile liquid (i.e., the portion of liquid which flows into liquid bridge region) on a particle divided by the total liquid content on particle $i$. We will see in the "Results" section that the mobility parameter itself is a function of the initial film height and the particle separation, but is invariant in time. For now, we simply use $\phi_{m, i}$ as a parameter that is constant during the filling process. Using a dimensionless filling rate parameter $a_{i}$ (which one can assume to be specific for each particle $i$ ) and the reference time scale $t_{\text {ref }}$, we finally arrive at the following differential equations for predicting the liquid content $L_{p, i}$ on each particle $i$ :

$$
\frac{d L_{p, i}}{d t}=\frac{-a_{i}}{t_{r e f}}\left(L_{p, i} \phi_{m, i}-\frac{V_{b}}{2}\right)
$$

This closure is linear in the unknown variables. Hence, an 410 analytical solution for the liquid bridge volume and the liquid 411 content remaining on the particles can be obtained. A simple 412 mass balance yields the governing equation for the volume 413 $V_{b, j}$ of bridge $j$ :

$$
\frac{d V_{b, j}}{d t}=-\sum_{i \in l_{j}} \frac{d L_{p, i}}{d t}
$$

Here $l_{j}$ is the list of particle indices that is in contact with 415 bridge $j$. We now rewrite these equations in dimensionless var- 416 iables, and apply them to a two-particle system. Together with 417 appropriate initial conditions, as well as the assumption that $a_{i} 418$ is a constant for a pair of particles sharing the same bridge, we 419 arrive at the following analytical solution:

$$
V_{b}^{+}=V_{b, 0}^{+}+C_{1} \frac{\frac{2 r_{1}}{a_{i}}+2 \phi_{m 1}}{r_{1}}\left(e^{r_{1} t^{+}}-1\right)+C_{2} \frac{\frac{2 r_{2}}{a_{i}}+2 \phi_{m 1}}{r_{2}}\left(e^{r_{2} t^{+}}-1\right)
$$

\begin{tabular}{|c|c|c|}
\hline Parameter & Value & Comment \\
\hline$\Delta t^{+}$ & $5 \times 10^{-3}$ & $\begin{array}{l}\text { Dimensionless } \\
\text { time step }\end{array}$ \\
\hline$\Delta h$ & $0.05-0.33$ & $\begin{array}{l}\text { Dimensionless } \\
\text { mesh resolution }\end{array}$ \\
\hline $\begin{array}{l}\text { Time derivative } \\
\text { scheme }\end{array}$ & Backward & $\begin{array}{l}\text { Second order, } \\
\text { implicit }\end{array}$ \\
\hline Laplacian scheme & $\begin{array}{c}\text { Gauss linear } \\
\text { corrected }\end{array}$ & $\begin{array}{l}\text { Unbounded, } \\
\text { second order, } \\
\text { conservative }\end{array}$ \\
\hline $\begin{array}{l}\text { Convection scheme } \\
\quad(\text { for } \mathrm{U})\end{array}$ & Gauss linear & $\begin{array}{l}\text { Unbounded, } \\
\text { second order }\end{array}$ \\
\hline $\begin{array}{l}\text { Convection scheme } \\
\quad(\text { for } \alpha)\end{array}$ & Gauss vanLeer & van Leer limiter \\
\hline
\end{tabular}

Table 1. Simulation Parameters and Numerical Schemes Used in the VoF Simulations 


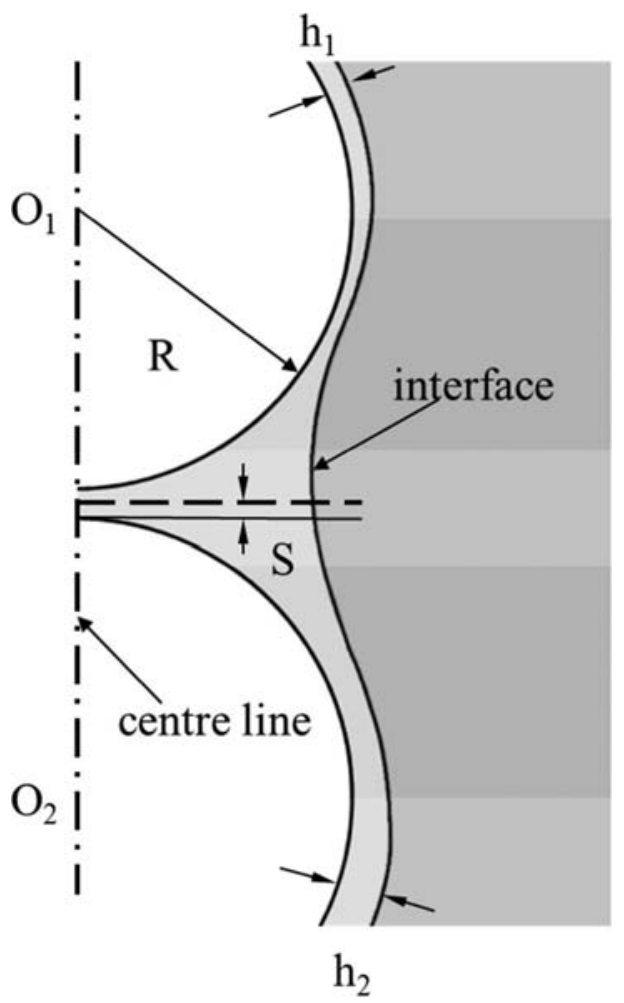

(a)

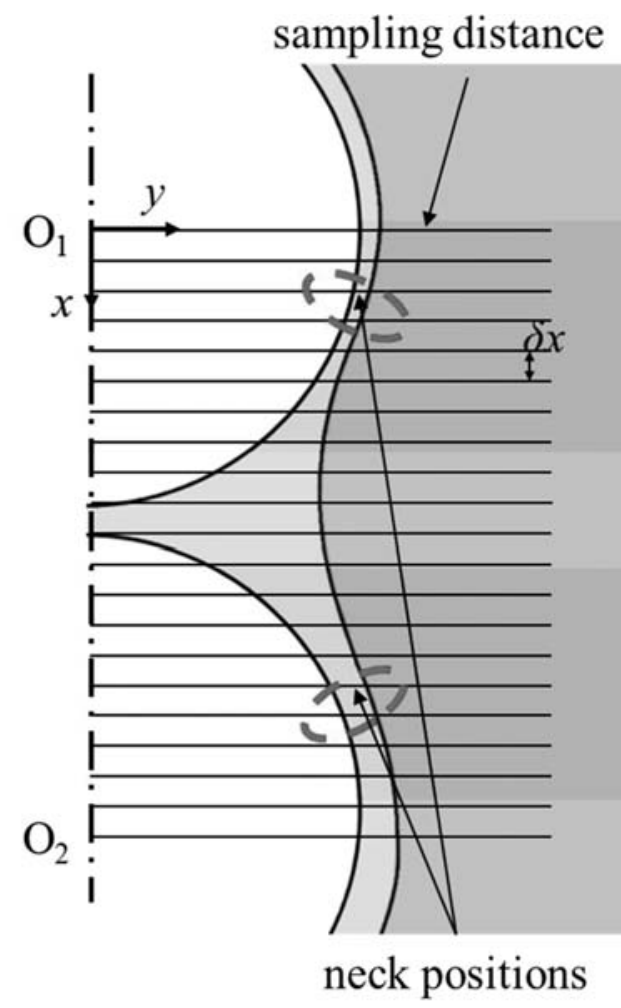

(b)

Figure 3. Sketch of (a) the gas-liquid interface between two particles, as well as (b) the sampling procedure used to detect the neck position.

[Color figure can be viewed in the online issue, which is available at wileyonlinelibrary.com.]

$$
\begin{array}{r}
L_{p 1}^{+}=L_{p 1,0}^{+}+\frac{C_{1}}{r_{1}}\left(e^{r_{1} t^{+}}-1\right)+\frac{C_{2}}{r_{2}}\left(e^{r_{2} t^{+}}-1\right) \\
L_{p 2}^{+}=L_{p 2,0}^{+}-C_{1} \frac{\frac{2 r_{1}}{a_{i}}+2 \phi_{m 1}+1}{r_{1}}\left(e^{r_{1} t^{+}}-1\right) \\
-C_{2} \frac{\frac{2 r_{2}}{a_{i}}+2 \phi_{m 1}+1}{r_{2}}\left(e^{r_{2} t^{+}}-1\right)
\end{array}
$$

where $r_{l}, r_{2}, C_{l}$, and $C_{2}$ are dimensionless coefficients that are detailed in Appendix A. Using this solution it is now straight forward to calibrate the model parameters (i.e., $a_{i}$ and $\phi_{m, i}$ ) with the results of our DNS (i.e., the time evolution of $L_{p}$ and $V_{b}$ ).

\section{Geometrical Bridge Volume}

In order to close the proposed model (see "Results" section), it is useful to define a reference bridge volume based on geometrical arguments. For such a geometrical bridge volume we have considered two types which are illustrated in Figure 4. Our goal is to study liquid bridge formation between particles having initially a different liquid content. Thus, it is useful to define a bridge volume based on the average (i.e., arithmetic mean) film height $h_{0}$ (see the definition in previous section "VoF Simulation Approach"). The expressions for the geometrical bridge volume detailed below need to be understood as the typical volume of a liquid bridge when making certain assumptions about its shape.

\section{Type I}

Type I (Figure 4a) is a simple definition based on the assumption that the initial bridge volume is that in the cap- shaped region of the particle. This definition is similar to the 442 idea of Shi and McCarthy ${ }^{46}$, which assumed that a fixed frac- 443 tion of the liquid present on the particle forms the bridge. This 444 previous definition of Shi and McCarthy would predict a linear 445 relationship between the bridge volume and the liquid film 446 thickness (and, to a first approximation, also with the liquid 447 content on each particle's surface). However, we argue that 448 this previous definition is unrealistic. The reason for this is 449 that the lateral extent (i.e., the length $b$ ) of the cap-shaped 450 region defined by the gas-liquid interface and the symmetry 451 plane between contacting particles is clearly a function of the 452 film thickness (see Figure 4, left panel). Hence, for the present 453 work we assume that the geometrical bridge volume of "type 454 I" is a nonlinear function of the liquid film thickness. Specifi- 455 cally, we assume that the bridge volume is equal to the red- 456 shaded cap-shaped region in Figure 4 (left panel). For this sit- 457 uation the liquid bridge volume can be calculated analytically: 458

$$
V_{b, g, I}=2 h_{0} b^{2} \pi
$$

with

$$
\frac{b}{R}=\left(1+h_{0}^{+}\right) \cos ^{-1}\left(1 /\left(1+h_{0}^{+}\right)\right)
$$

\section{Type II}

Type II considers another shape of the liquid bridge, which 461 is shown in Figure 4b. Here, we take the separation distance of 462 the particle into account, implying that the type II geometrical 463 bridge volume is more realistic for large separations. Specifi- 464 cally, we assume that the liquid in the overlapping regions 465 (given by the assumed spherical shape of the liquid films on 466 


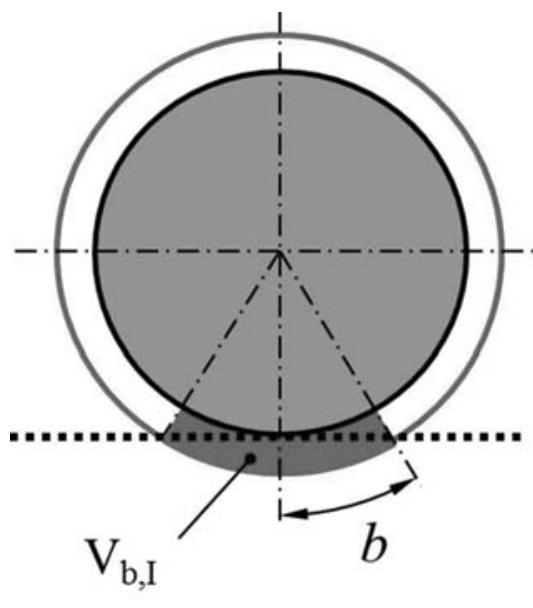

(a)

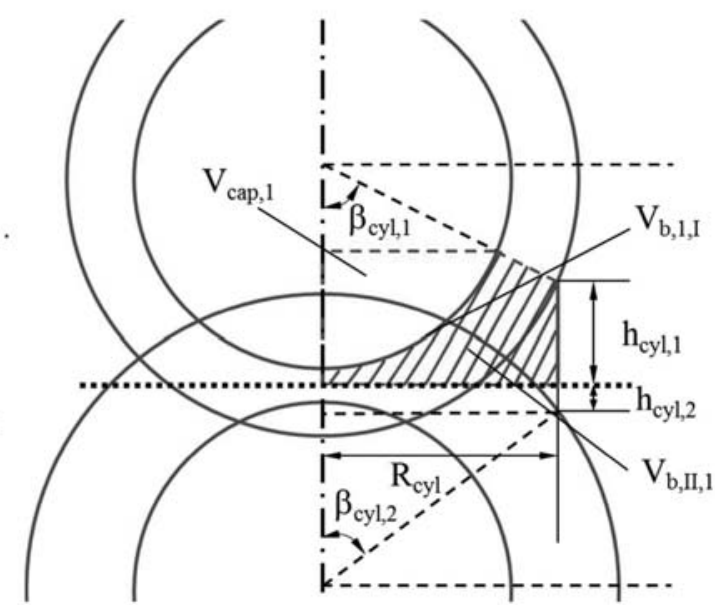

(b)

Figure 4. Geometrical bridge volume: (a) type I (the dashed line indicates the symmetry plane of two contacting particles), (b) type II.

[Color figure can be viewed in the online issue, which is available at wileyonlinelibrary.com.]

the particle) must be laterally displaced when the particles approach each other. This liquid is assumed to flow into a ring-shaped region. One can then compute the liquid bridge volume from the red shaded area (see Figure 4, right panel). Specifically, the volume of the type II bridge geometry can be calculated as follows:

$$
V_{b, g, I I}=V_{b, i, I}-V_{\text {cap }}
$$

The evaluation of the above equation involves lengthy expressions, and is detailed in Appendix B. Unfortunately, this evaluation also requires an iterative procedure, making it less attractive for direct evaluation during large-scale DEM-based simulation.

We next compare these two types of models for the geometrical bridge volume for two situations: in situation A we increase the liquid content (i.e., the initial film heights), but assume contacting particles (i.e., zero separation between particles). Results for this situation are shown in Figure 5, which shows the dimensionless bridge volume as a function of the $L_{p 0}^{+}$, where $L_{p 0}^{+}$is dimensionless form of average amount of initial liquid content of the two particles and defined by $L_{p 0}^{+}=\frac{4}{3} \pi\left(\left(R+h_{0}\right)^{3}-R^{3}\right) / R^{3}=\frac{4}{3} \pi\left(\left(1+h_{0}^{+}\right)^{3}-1\right)$. We observe that for both types of definitions the bridge volume increase with the liquid content nonlinearly. Note, that in the definition of Shi and McCarthy ${ }^{46}$ the bridge volume increases linearly. Furthermore, we see that the two curves for type I and type II nearly overlap, and that type II predictions are slightly larger than that of type I. Thus, the two curves agree well with each other for thin initial films, while they do not agree with each other for thicker films. Since the definition of the type II model at zero separation is close to that of type I, this is expected and explained as follows: the type II model takes into account that the laterally displaced liquid forms a bridge with a certain height $h_{\mathrm{cyl}, l}$ and $h_{\mathrm{cyl}, 2}$ (see Figure 4, right panel). Consequently, a certain amount of the liquid on the particle (in addition to that accounted for in the type I model) is considered to be in the bridge. Thus, when using the type II definition, the (geometrical) bridge volume is somewhat larger compared to that predicted by type I. In summary, we see that the type I approximation is appropriate for thin films and at zero separa- tion, while type II should be considered for all other 505 situations.

Situation B is now investigated to demonstrate the effect of 507 the particle-particle separation distance on the predicted (geo- 508 metrical) bridge volume. Specifically, a certain (constant) ini- 509 tial film height was assumed for both types of models, and the 510 separation between particles was varied. Results are illustrated 511 in Figure 6. One can observe that the prediction of the type I 512 F6 model is not affected by the separation distance. However, for 513 the type II model, the geometrical bridge volume remarkably 514 decreases when the separation distance increases. Also, the 515 type II model predicts a somewhat larger bridge volume (com- 516 pared to type I) at zero separation as it should be. In summary, 517 the type II model is more realistic, and we will demonstrate in 518 following that our results (based on DNS) are very close to the 519 predictions of this model.

Figure 7 further illustrates how the separation distance $521 \mathrm{~F} 7$ affects the prediction of the (geometrical) bridge volume when 522 using the type II model. As can be seen from Figure 7 the 523

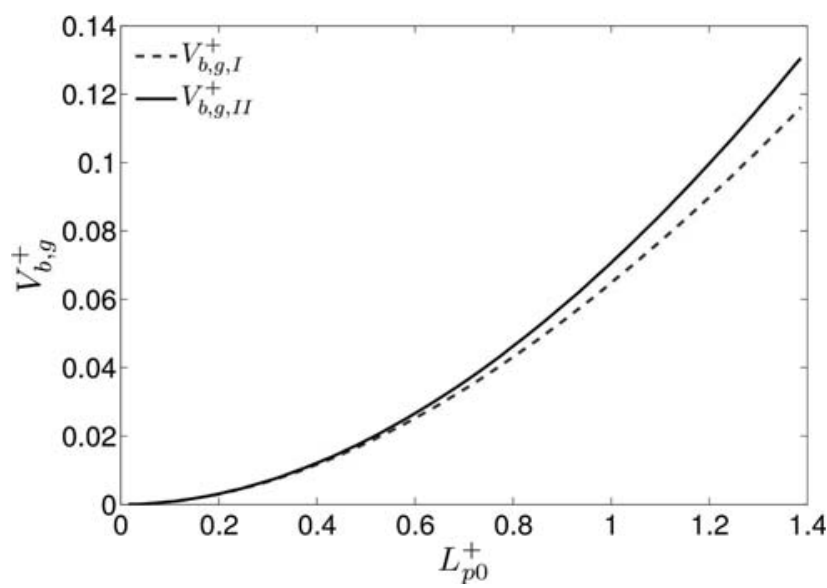

Figure 5. Comparison of the type I and type II model for computing the geometrical bridge volume in situation $\mathrm{A}$ : increasing liquid content on the particles at $S^{+}=0$.

[Color figure can be viewed in the online issue, which is available at wileyonlinelibrary.com.] 


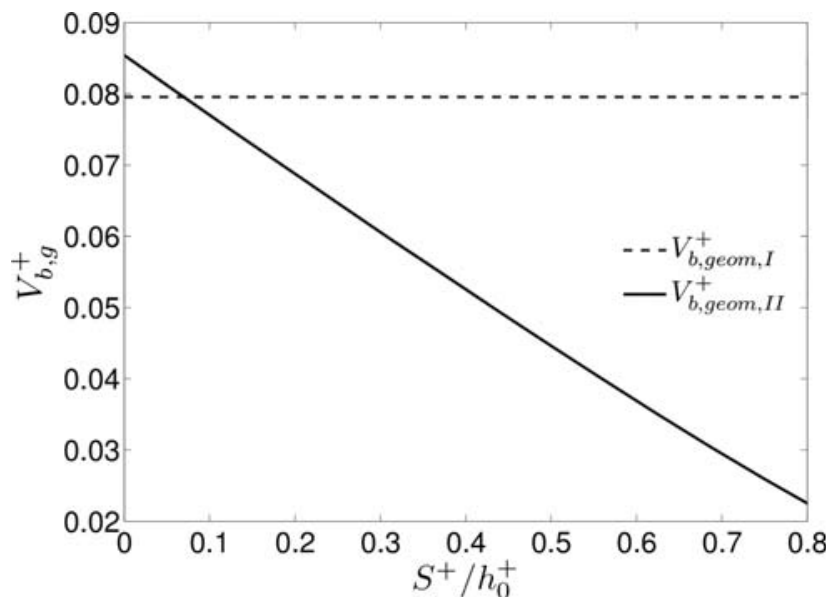

Figure 6. Comparison of the type I and type II model for computing the geometrical bridge volume in situation B: fixed initial film height $h_{0}^{+}=0.08$ and variation of the separation distance.

[Color figure can be viewed in the online issue, which is available at wileyonlinelibrary.com.]

bridge volume increases monotonically, but nonlinearly, with increasing (initial) liquid content for every choice of separation distance. In the situation of zero separation the largest bridge volume is predicted. Also, it can be observed that the bridge volume decreases almost linearly with increasing separation distance, finally approaching zero for $S^{+} / h_{0}^{+}=1$ as it should be. The physical interpretation of this fact is that for the situation in which the separation equals the initial film thickness, the overlapping region of thin films between two particles vanishes. Thus, the geometrical bridge volume becomes zero. In summary, the type II model shows the correct behaviour for a variety of limiting cases. Hence we argue that it is physically more relevant compared to previous work.

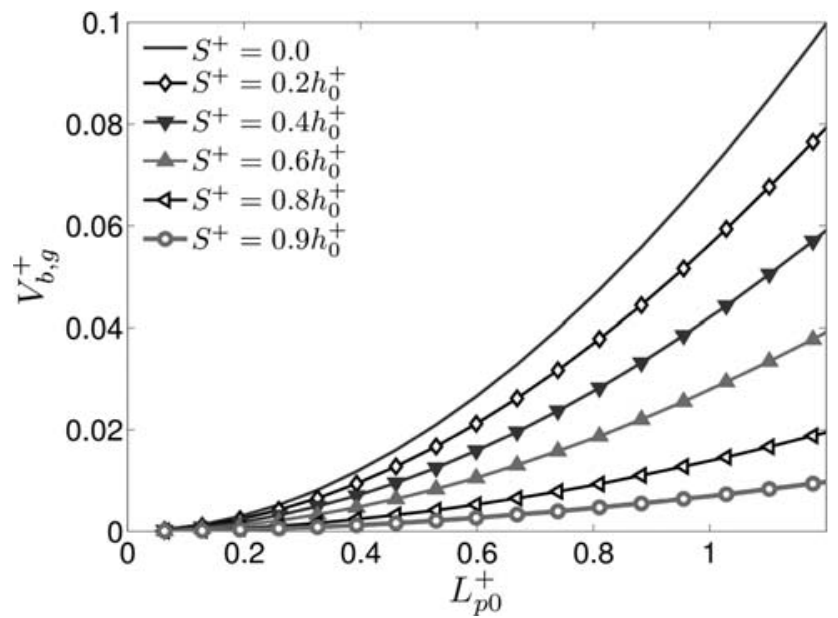

Figure 7. Effect of the separation distance on the bridge volume as a function of the initial liquid content (type II model).

[Color figure can be viewed in the online issue, which is available at wileyonlinelibrary.com.]

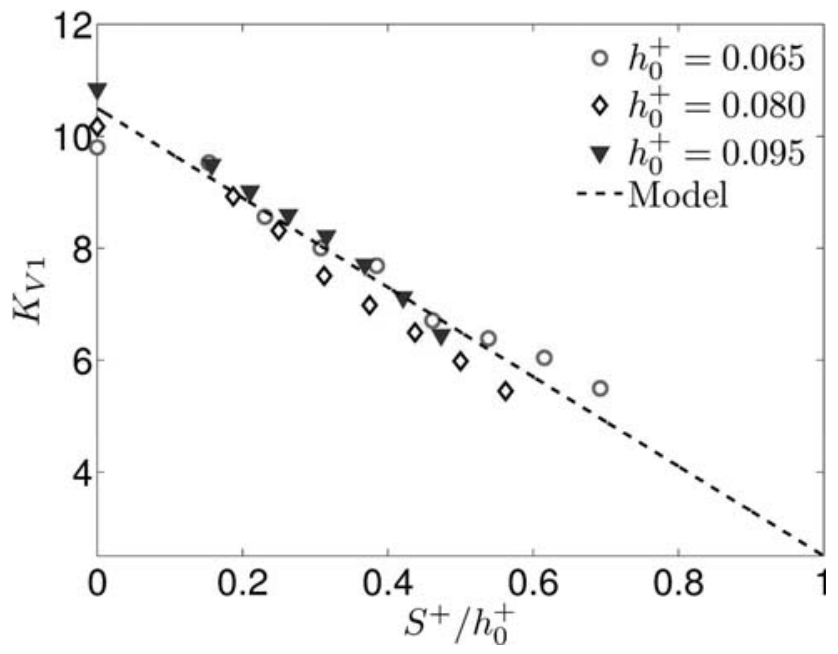

Figure 8. Normalized initial bridge model $K_{V_{1}}$ vs. normalized separation distance.

[Color figure can be viewed in the online issue, which is available at wileyonlinelibrary.com.]

\section{Results}

Early stage model (stage I)

The initial bridge forms very quickly, and the inertia of the 540 fluid may play a certain role. Inertia is difficult to model due 541 its inherent nonlinearity. We hence define a fixed initial bridge 542 volume for "early times." Specifically, we have chosen the 543 early stage to end after one reference viscous time scale, that 544 is, at $t^{+}=1$.

We now attempt to model the initial bridge volume by 546 defining the variable $K_{V l}$, which is the ratio of the simulated 547 (total) initial bridge volume $\left(V_{b, 0}^{+}\right.$is the liquid bridge volume 548 at $\left.t^{+}=1\right)$ and the average initial film height $h_{0}^{+}$to the power 549 of some exponent $n$ :

$$
K_{V 1}=\frac{V_{b, 0}^{+}}{\left(h_{0}^{+}\right)^{n}}
$$

This definition is based on the simple idea that the initial 551 bridge volume is only a function of the (initial) film height, 552 similar to the idea we used to define the geometrical bridge 553 volume of type I. We see from Figure 8 that when choosing 554 F8 $n=1.5$ we can collapse our DNS results for a variety of film 555 thicknesses with the expression:

$$
K_{V 1}=-8.0\left(\frac{S^{+}}{h_{0}^{+}}\right)+10.5
$$

Thus, we see that we can obtain a reasonable collapse of our 557 data on a straight line for this choice of $n$ and for $S^{+} / h_{0}^{+}<0.7$. 558 We also note that (1) the normalized bridge volume linearly 559 decreases with increasing separation distance, and (2) that the 560 bridge volume is a super-linear function of the film height. 561 The former fact is in agreement with the type II geometrical 562 bridge volume (refer to "Geometrical Bridge Volume" sec- 563 tion). The latter again stresses the fact that the assumption of 564 Shi and McCarthy ${ }^{46}$ that liquid is "harvest" from a fixed (area) 565 fraction of the particle surface is not supported by our results. 566 A model based on "harvesting" from a fixed (area) fraction of 567 the particle surface would result in a linear increase of the 568 bridge volume with film height, that is, $n=1$. Clearly, this is 569 not supported by our DNS data.

.




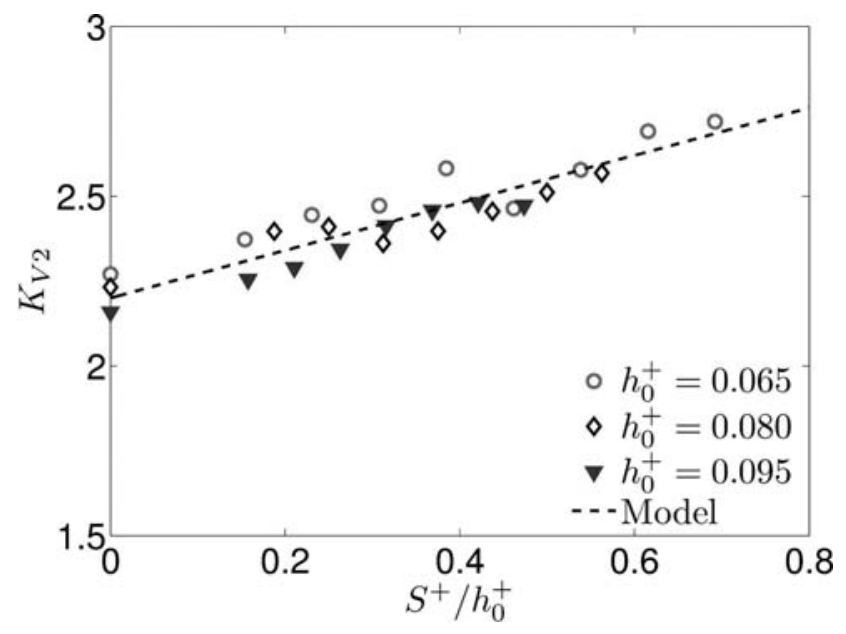

Figure 9. Initial bridge model, $K_{V 2}$ vs. normalized separation distance.

[Color figure can be viewed in the online issue, which is available at wileyonlinelibrary.com.]

In summary, our model for $K_{V 1}$ could already be used to compute $V_{b, 0}^{+}$for a collision involving two wet particles using Eq. 15. However, we next aim at using the geometrical bridge volume of type II to normalize the calculated initial bridge volume. We do this since this model accounts for the effect of the separation distance on the bridge volume, and we expect that a normalization with this geometrical bridge volume yields a variable that is independent of $S^{+}$.

Specifically, we define the variable $K_{V 2}$ as the ratio of the measured initial bridge, the geometrical bridge volume calculated using the type II model (refer to "Type II" section), and a function of $h_{0}^{+}$:

$$
K_{V 2}=\frac{V_{b, 0}^{+}}{V_{b, g, I I}^{+}\left(h_{0}^{+}\right)^{n}}
$$

Again, $n$ is a parameter that is used as an exponent of the initial film height, and helps to collapse all data into a single curve. We expect that $n$ is close to zero, that is, that the geometrical bridge volume based on the type II model is sufficient to account for any effects due to the film height. The results of our analysis are displayed in Figure 9, which illustrates that the variable $K_{V 2}$ is independent of the average film height, when choosing $n=0.2$. For the $K_{V 2}$ model, we also suggest a linear relationship between the normalized liquid bridge volume and the separation distance:

$$
K_{V 2}=0.7\left(\frac{S^{+}}{h_{0}^{+}}\right)+2.2
$$

In addition we note that assuming $n=0$, that is, using a normalization purely based on the geometrical bridge volume, would be also a good approximation (data not shown). Interestingly, we find that $K_{V 2}\left(h_{0}^{+}\right)^{n} \approx 1.3$ for zero separation, indicating that the geometrical bridge volume of type II is indeed a good approximation of the initial bridge volume. Also, we find that $K_{V 2}$ increases with increasing separation, indicating that the geometrical bridge volume of type II overcompensates the decrease of the bridge volume. Thus, our DNS data suggest that the bridge volume is systematically larger for $S^{+}>0$ than that based on geometrical arguments. The exact reason for this is could not be isolated. However, it is clear that in the DNS the deformation of the gas-liquid interface is also taken into account. We speculate that this 606 deformation leads to an additional lateral shift of the neck 607 position (in addition to the shift caused by to the displaced 608 fluid), which is more pronounced for larger separations. 609

In summary, the model for $K_{V 2}$ presented above, together 610 with its definition, and the geometrical bridge volume (type II) 611 can be used to compute the bridge volume at $t^{+}=1$. We next 612 focus on the calibration of the parameters in the proposed 613 bridge viscous filling model (see section "Proposed Model for 614 Liquid Bridge Filling"), that can be used to predict the time 615 evolution of the liquid bridge after this point in time.

Viscous filling stage model (stage II)

We start with looking at the time evolution of bridge vol- 618 ume and liquid present on the particle for the situation of zero 619 separation start by taking one case for example (see Figure 620 $10)$. We obtain the following parameter set $\phi_{m l}=0.49,621 \mathrm{~F} 10$ $\phi_{m 2}=0.38$, and find that mobility parameter of particle 1 is 622 somewhat larger than that of particle 2 (particle 1 initialized 623 with less liquid content than particle 2). Furthermore, we find 624 that the dimensionless filing rate coefficient $a_{i}$ (i.e., the inverse 625 of a dimensionless filling time scale) is approximately 0.025. 626 This value fits all our data reasonably well, and hence we 627 accept $a_{i}$ to be a universal constant from now on.

Results in Figure 11 are also obtained for the zero separation $62 \mathrm{~F} 11$ case, however, for a different combination of film thicknesses. 630 The mobility parameters for this case are $\phi_{m l}=0.45$ and 631 $\phi_{m 2}=0.43$, respectively. By comparing Figures 10 and 11, we 632 observe that the trend of liquid transport between two particles 633 are similar, and the model approximates the filling process rea- 634 sonably well. As expected, the case with the larger (average) 635 initial film height yields a larger liquid bridge volume.

636

Further testing of our model for other combinations of 637 thickness reveals that our model is indeed able to describe the 638 filling process well. As we can see from Figure 12, larger film 63 F12 height always leads to larger bridge volume, which is obvious. 640 We also can see that the filling process levels off after about 641 50 dimensionless time units. This is also suggested by the 642 inverse of the constant $a_{i}$, which has been fixed before. 643

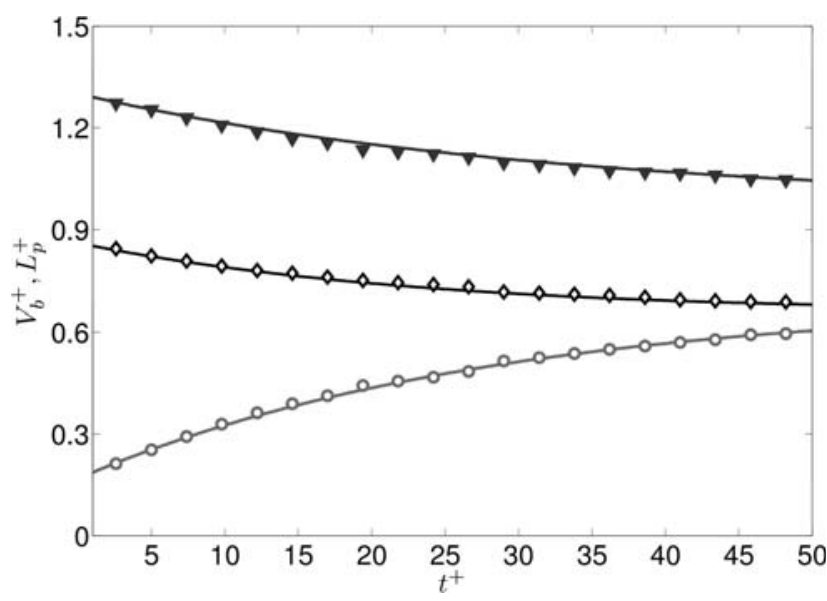

Figure 10. Fitted model (lines) vs. DNS data (symbols) over time for $S^{+}=0, h_{1}^{+}=0.07$, and $h_{2}^{+}=$ 0.1 .

Red circles: liquid bridge volume $\left(\boldsymbol{V}_{b}^{+}\right)$; Black diamonds: liquid content on particle $1\left(L_{P 1}^{+}\right)$; Blue triangles: liquid content on particle $2\left(L_{P_{2}}^{+}\right)$. [Color figure can be viewed in the online issue, which is available at wileyonlinelibrary.com.] 


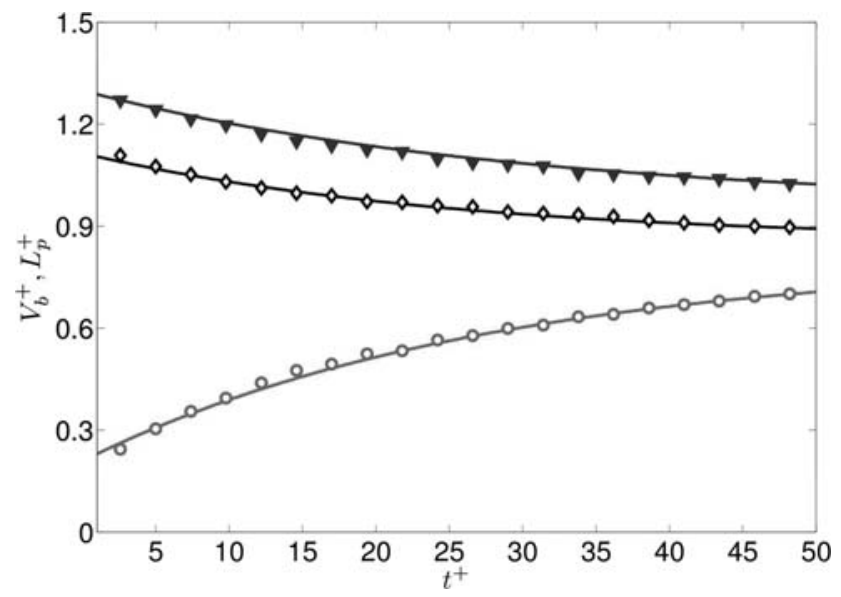

Figure 11. Fitted model (lines) vs. DNS data (symbols) over time for $S^{+}=0, h_{1}^{+}=0.09$ and $h_{2}^{+}=0.1$.

Red circles: liquid bridge volume $\left(V_{b}^{+}\right)$; Black diamonds: liquid content on particle $1\left(L_{P 1}^{+}\right)$; Blue triangles: liquid content on particle $2\left(L_{P_{2}}^{+}\right)$. [Color figure can be viewed in the online issue, which is available at wileyonlinelibrary.com.]

However, the filling process has not completely stopped at $t^{+}=50$. Indeed, we find that when running the simulation for a longer duration the filling process will end at some point due to the rupture of the film on one of the two particle surfaces. We will discuss this peculiar behavior later (see section "Film Rupture and Grid Refinement").

Considering now separations larger than zero, we again see that the model is able to approximate the DNS data well (see Figure 13 , for $S^{+}=0.045, h_{1}^{+}=0.09$, and $h_{2}^{+}=0.1$ ). However, we find that the parameters $\phi_{m 1}$ and $\phi_{m 2}$ change. Specifically, the value of the mobility parameters is now $\phi_{m l}=0.42$ and $\phi_{m 2}=0.39$, respectively. Thus, the mobility of the liquid on the particles becomes smaller for $S^{+}>0$ compared to the case with $S^{+}=0$. Our interpretation of this fact is that less liquid is mobile to flow into the bridge when the particles have a certain separation. Again, we can see from Figure 14 that larger initial film height causes larger liquid bridge volume, as expected.

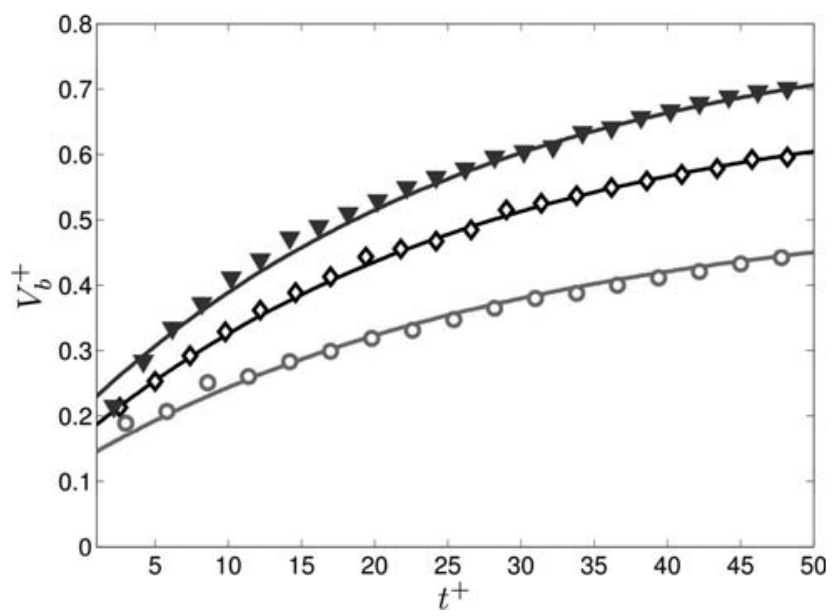

Figure 12. Liquid bridge volume over time: fitted model (lines) vs. DNS data (symbols), $\mathrm{S}^{+}=0$.

Red circles: $h_{0}^{+}=0.075$; Black diamonds: $h_{0}^{+}=0.085$; Blue triangles: $h_{0}^{+}=\mathbf{0 . 0 9 5}$. [Color figure can be viewed in the online issue, which is available at wileyonlinelibrary.com.]

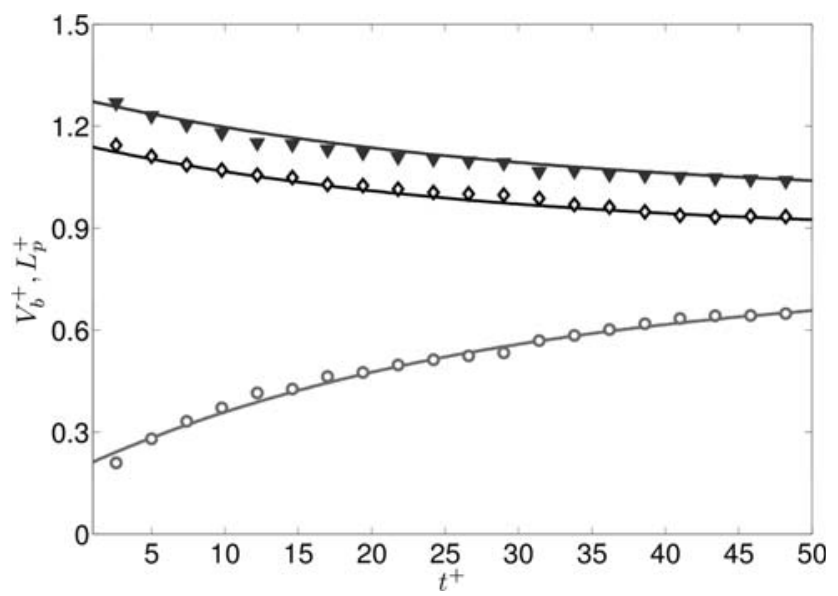

Figure 13. Fitted model (lines) vs. DNS data (symbols) over time for $S^{+}=0.045, h_{1}^{+}=0.09$ and $h_{2}^{+}=0.1$.

Red circles: liquid bridge volume $\left(V_{b}^{+}\right)$; Black diamonds: liquid content on particle $1\left(L_{P_{1}}^{+}\right)$; Blue triangles: liquid content on particle $2\left(L_{P_{2}}^{+}\right)$. [Color figure can be viewed in the online issue, which is available at wileyonlinelibrary.com.]

We now aim on demonstrating that our model is able to rep- 661 resent data for a variety of dimensionless initial film heights. 662 Since the key parameter that is influenced by the film height is 663 the mobility (i.e., $\phi_{m, i}$ ), we have collected these parameters for 664 a large set of separation distances and dimensionless initial 665 film heights. We now make an attempt of modeling $\phi_{m, i}$ by 666 first computing an average mobility $\phi_{m}$ (see Eq. 18), that is 667 simply defined as the arithmetic mean of the motilities of the 668 particles in contact. We now look at the trends of this average 669 parameter as a function of the initial average film height. As 670 we can see from Figure $15, \phi_{m}$ changes linearly with the aver- 67 F15 age initial film height for zero separation. Specifically, we 672 obtain the following relationship for $\phi_{m}$ :

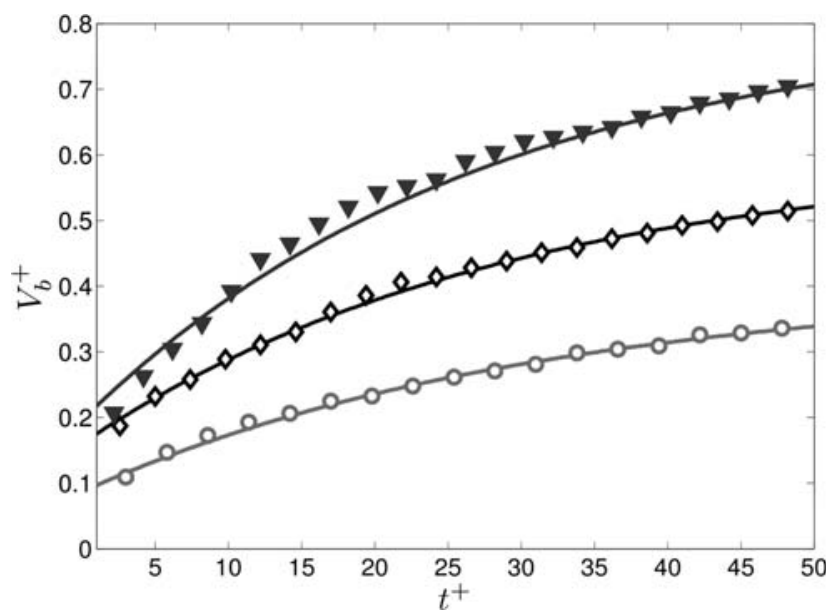

Figure 14. Combined effect of film height and separation distance: liquid bridge volume over time: fitted model (line) vs. DNS data (symbols).

Red circles: $S^{+}=0.045$ and $h_{0}^{+}=0.065$; Black diamonds: $S^{+}=0.025$ and $h_{0}^{+}=0.08 ;$ Blue triangles: $S^{+}=0.035$ and $h_{0}^{+}=0.095$. [Color figure can be viewed in the online issue, which is available at wileyonlinelibrary.com.] 


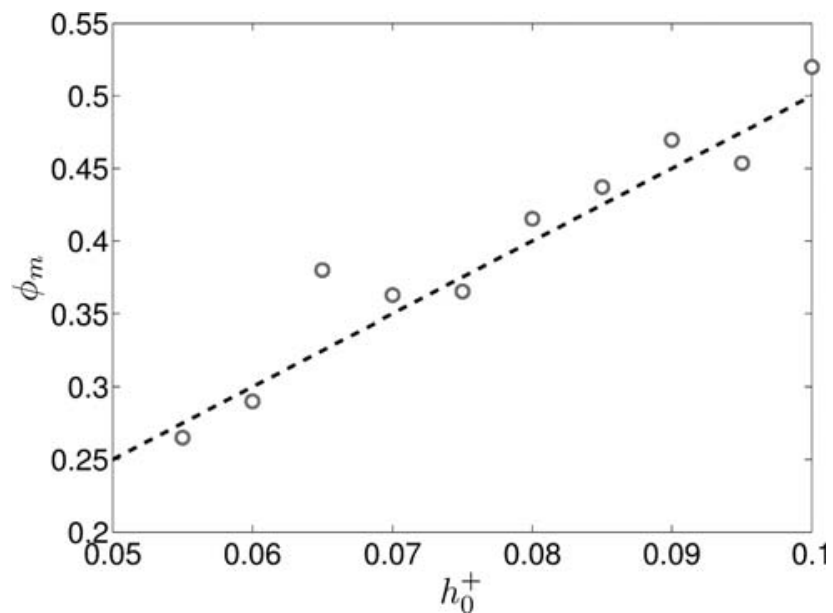

Figure 15. $\phi_{m}$ as a function of $h_{0}^{+}$for a separation of $S^{+}=0$.

[Color figure can be viewed in the online issue, which is available at wileyonlinelibrary.com.]

$$
\begin{gathered}
\phi_{m}=\frac{\phi_{m 1}+\phi_{m 2}}{2} \\
\phi_{m}=5 h_{0}^{+}
\end{gathered}
$$

For the difference in the mobilities, denoted as $\Delta \phi_{m}=$ $\left|\phi_{m 1}-\phi_{m 2}\right|$ and illustrated in Figure 16, we find the following approximation:

$$
\Delta \phi_{m}=2.9 \Delta h_{0}^{+}
$$

Next, we aim at correlating $\phi_{m}$ with the separation distance. Specifically, we consider data sets for variations in the initial film height and separation distance shown in Figure 17. Again, we can collapse all data for different initial film heights when normalizing it with some function of the initial film height. Specifically, we define the variable $K_{\phi m}$ to be

$$
K_{\phi_{m}}=\frac{\phi_{m}}{5 h_{0}^{+}}
$$

Based on the data shown in Figure 17, we find the following relationship for $K_{\phi m}$ :

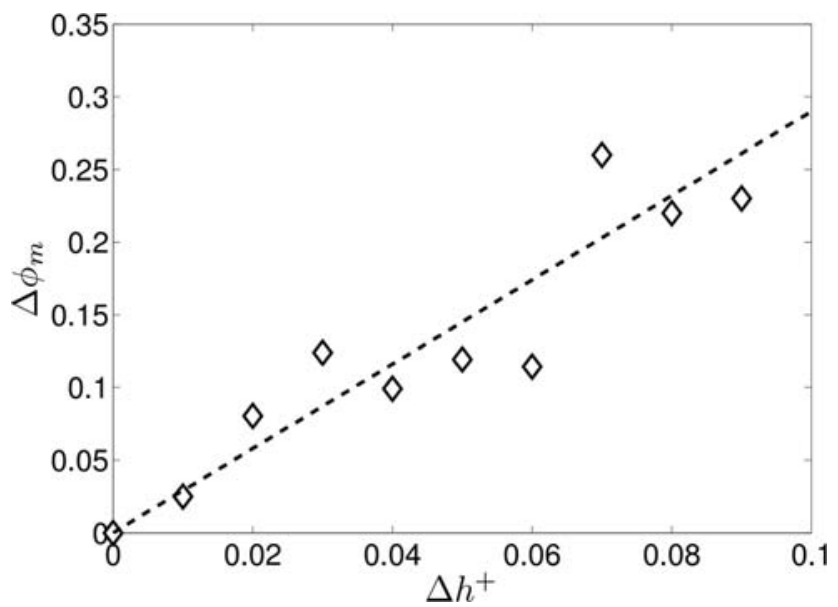

Figure 16. $\Delta \phi_{m}$ as a function of the dimensionless difference of the film heights.

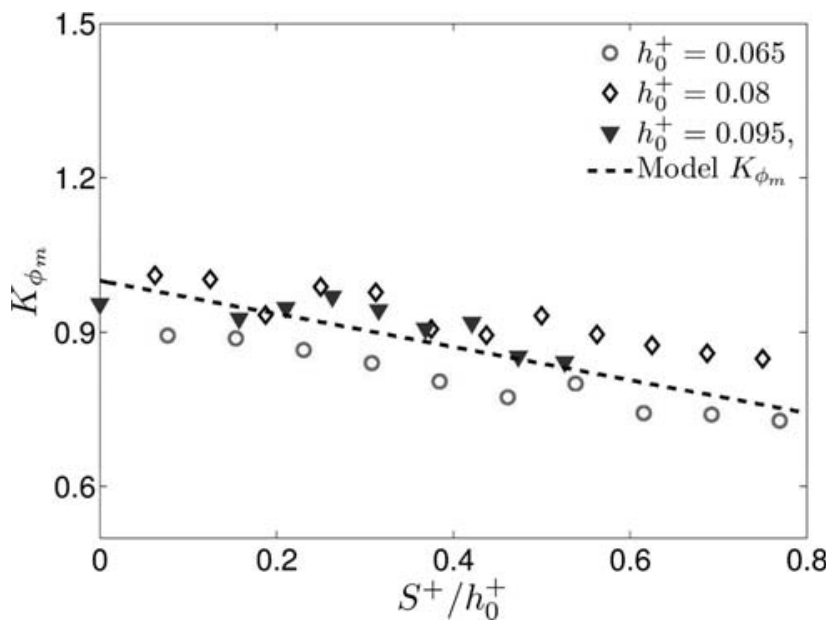

Figure 17. $K_{\phi_{m}}$ as a function of the normalized separation distance for contacting and noncontacting particles.

[Color figure can be viewed in the online issue, which is available at wileyonlinelibrary.com.]

$$
K_{\phi_{m}}=1-0.32\left(\frac{S^{+}}{h_{0}^{+}}\right)
$$

The meaning of these relationships is that the average 685 mobility of the liquid on the particle surfaces systematically 686 decreases with increasing separation distance. The physical 687 interpretation of this fact is that smaller bridges (implied by 688 larger separation distances and constant overall liquid content) 689 simply allow proportionally less liquid to flow from the parti- 690 cle surface into the bridge. In contrast, at small separation dis- 691 tances, and hence larger bridges, a larger fraction of the 692 particle's surface area is connected to the bridge, and hence 693 the mobility parameter is large.

\section{Film rupture and grid refinement}

We also show some interesting findings which we observe 696 for very long simulation times and thin films: as can be seen in 697 Figure 18 the film ruptures at the neck position for sufficiently $69 \mathrm{~F} 18$ long time. Specifically, we observe that for thin films the film 699 ruptures on the particle with initially less liquid (i.e., particle 700 1). This means that the bridge filling process stops after a cer- 701 tain time, which is also the case for the proposed model. 702 Unfortunately, we cannot accurately predict the rupturing pro- 703 cess, simply because the film at the rupturing point must 704 become thinner than the (finite) grid resolution. It is therefore 705 essential how the grid resolution affects the film rupturing 706 event. This is discussed next.

We start our investigation of the effect of grid refinement 708 by defining a dimensionless grid size $\Delta h$. Specifically, we 709 choose $\Delta h=\Delta x / h_{l}$, where $\Delta x$ is the mesh size and $h_{l}$ is the 710 initial film height of the particle with the lower amount of liq- 711 uid on its surface. As can be seen from Figure 19, the grid 71 F19 refinement affects the filling process only negligibly, with the 713 largest deviations observed for long times, that is, $t^{+}>100.714$ As can be seen in the Figure for the case of a grid size of 715 $\Delta h=0.17$ (blue circles) film rupture is observed at $t^{+}=175.716$ However, in case of a larger grid resolution, that is, $\Delta h=0.12717$ (black diamonds), we do not observe film rupture and the fill- 718 ing process continues until the simulation was terminated. 719 Therefore, grid refinement plays an important role for 720 


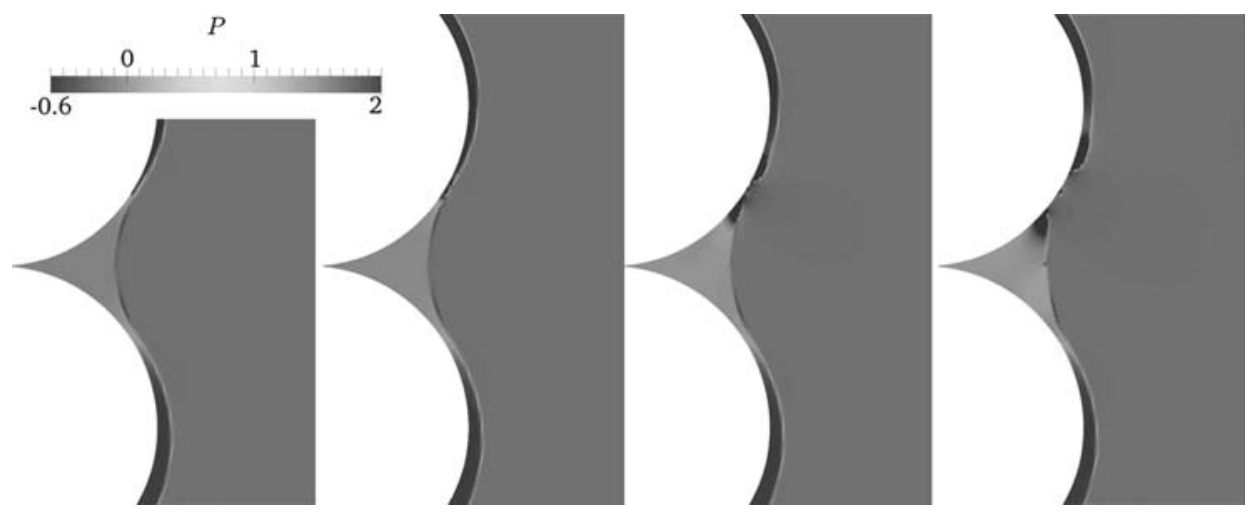

Figure 18. Pressure distribution before and after the film rupture moment $t^{+}=220, s^{+}=0, h_{1}^{+}=0.06, h_{2}^{+}=0.10$ (the rupture time scale is small, that is, below $t^{+}<1$ ).

[Color figure can be viewed in the online issue, which is available at wileyonlinelibrary.com.]

predicting the final rupturing process. Moreover, it can be observed that a finer mesh yields a rather smooth curve, in contrast to the results for the coarser grid in which the bridge volume appears to fluctuate. This is again caused by the more challenging detection of the neck position in the case of a (comparably) coarse grid.

We have observed in our simulations that the film rupturing phenomena occurs on particles with less liquid. The obvious reason for film rupturing in the simulation is inadequate resolution of the liquid film in the neck region. The thinning of the film there is caused by the pressure difference over the neck region, which drives the flow of liquid into the bridge: due to the complex shape of the gas-liquid interface in this region, the pressure changes in a nonlinear way in the neck region. The pressure distribution is such that more liquid exits the neck region than can flow from the film toward the neck. This leads to a thinning of the film, and once the film thickness is in the order of the grid resolution, it will rupture. We hence must limit the applicability of our model to situations well before the rupture event occurs in the simulation, that is, to $t^{+}<100$.

\section{Reynolds number and density ratio effects}

In order to further investigate the model's ability to reflect various real-world situations, we checked the effect of the Reynolds number on the filling process. Therefore, we have chosen a situation with rather thin films (i.e., $h_{1}^{+}=0.04$, $\left.h_{2}^{+}=0.08, S^{+}=0.02\right)$. We choose $R e=1, R e=100$, and $R e=10,000$ for investigating Reynolds number effects.

As we can see from Figure 20, the points at which film rupture occurs are almost identical. Also, we observe that larger Reynolds number lead to an earlier film rupturing event (see Figure 21). However, the filling process of the bridge is not substantially affected by the Reynolds number. Hence, we draw the conclusion that the Reynolds number plays a negligible role for the bridge filling process, at least in the range of parameters we have investigated.

Finally, we have investigated the effect of the density ratio on the liquid bridge filling process (see our results in Figure 22 for the density ratios of $\rho=10$, and $\rho=1000$ ). The density ratio is a critical parameter for the numerical simulation, since simulations with a smaller density ratio are typically easier to conduct. As can be seen from Figure 22 the density ratio has little effect on the liquid bridge filling process as long as $\rho \geq 10$, and the rupturing event is delayed by about five dimensionless time units in the case of the low density ratio that has been investigated. Therefore, we can safely neglect 765 effects due to the ambient gas density when considering bridge 766 filling in gas-liquid-particle systems.

\section{Initial bridge shape effects}

In this section we summarize data on the effect of the ini- 769 tial shape of the liquid bridge on the bridge filling process. 770 As shown in Figure 23, four types of initial bridge shapes 77 F23 have been investigated, that is, the standard cylinder (denoted 772 as "cylinder"), no bridge ("none"), a too large cylinder 773 ("large cylinder"), i.e., the radius is $20 \%$ larger than standard 774 cylinder, and a smooth curve in the form of a circle 775 ("circle"). The results are shown in Figure 24, and we $77 \mp 24$ observe that the initial liquid bridge shape has generally a 777 small effect on the filling process, except for the situation 778 "large cylinder". For this situation significantly more liquid 779 is in the bridge, however, the qualitative behavior of the fill- 780 ing process is preserved. Hence, we conclude that as long as 781 the bridge is initialized with a realistic shape (i.e., a cylinder 782 containing the displaced fluid), the effect of the exact initial 783 shape is in the order of $3.3 \%$ between "none" and "cylinder", 784 and $3.9 \%$ between "circle" and "cylinder".

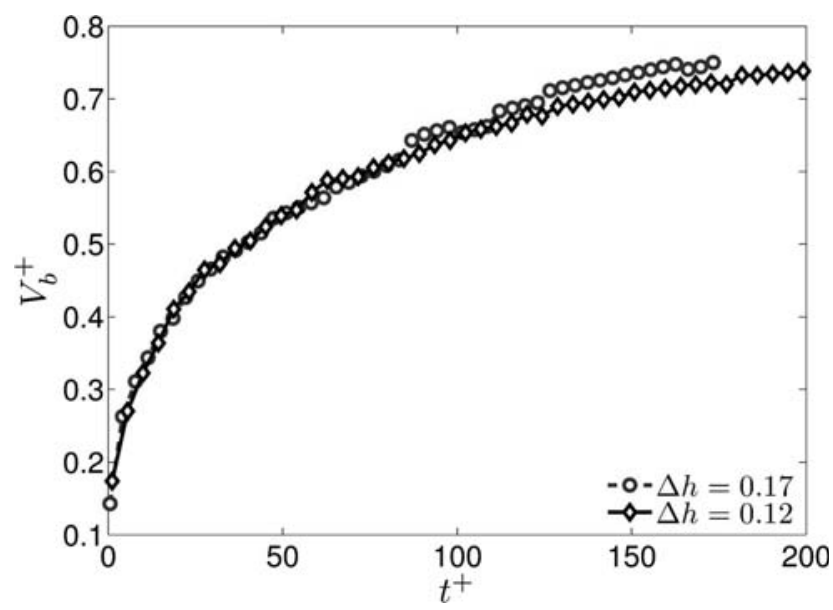

Figure 19. Grid refinement effects on the liquid bridge filling, $h_{1}^{+}=0.04, h_{2}^{+}=0.08, s^{+}=0.02$.

[Color figure can be viewed in the online issue, which is available at wileyonlinelibrary.com.] 


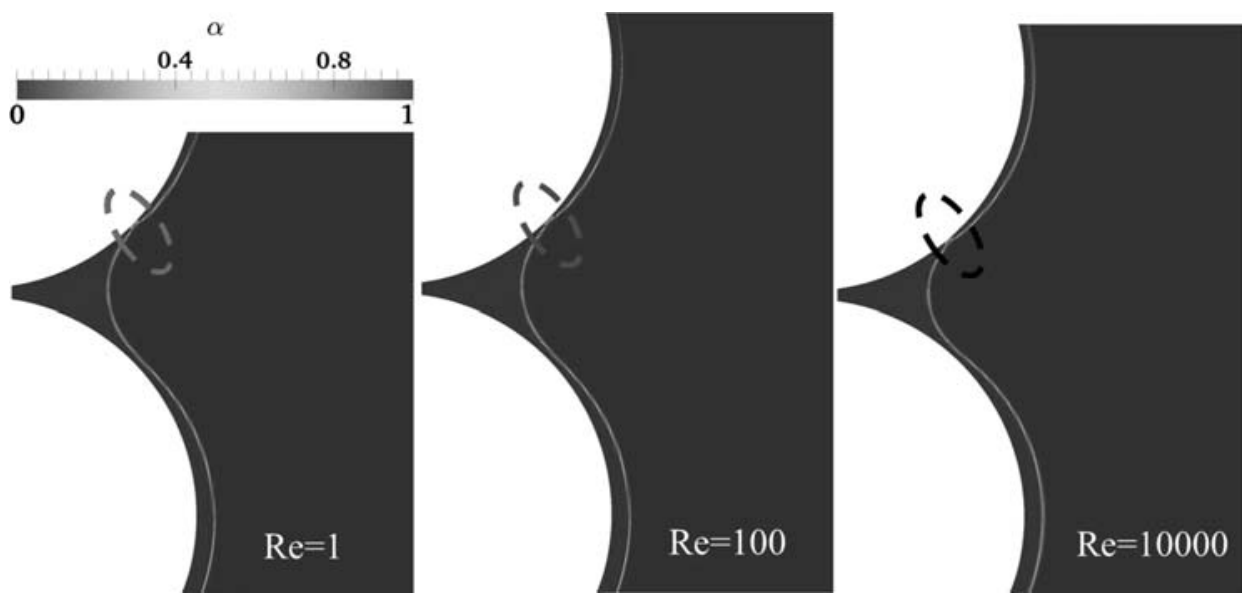

Figure 20. Liquid film distribution at the rupture moment, $h_{1}^{+}=0.04, h_{2}^{+}=0.08, S^{+}=0.02$.

[Color figure can be viewed in the online issue, which is available at wileyonlinelibrary.com.]

\section{Discussion}

In our study, we investigate the liquid bridge and drainage process of liquid adhering to two wet particles based on key dimensionless parameters. We provide a model for the prediction of dynamic liquid-bridge formation between two particles by assuming that the filling rate of the liquid bridge is not affected by the particles' relative motion. Thus, we assume a quasi-static situation in which particles do not move, but only liquid is mobile to flow into the bridge. We next perform a time scale analysis to probe situations for which such a quasistatic assumption is appropriate.

\section{Particle interaction time scales}

A key question that could not be answered in the current article is what happens in case the particles move relative to each other, and hence the above mentioned quasi-static assumption breaks down. In order to do so, we must identify the limits of the models proposed in the current work. Specifically, there are two criteria that need to be satisfied to accept the assumption of zero relative particle velocity:

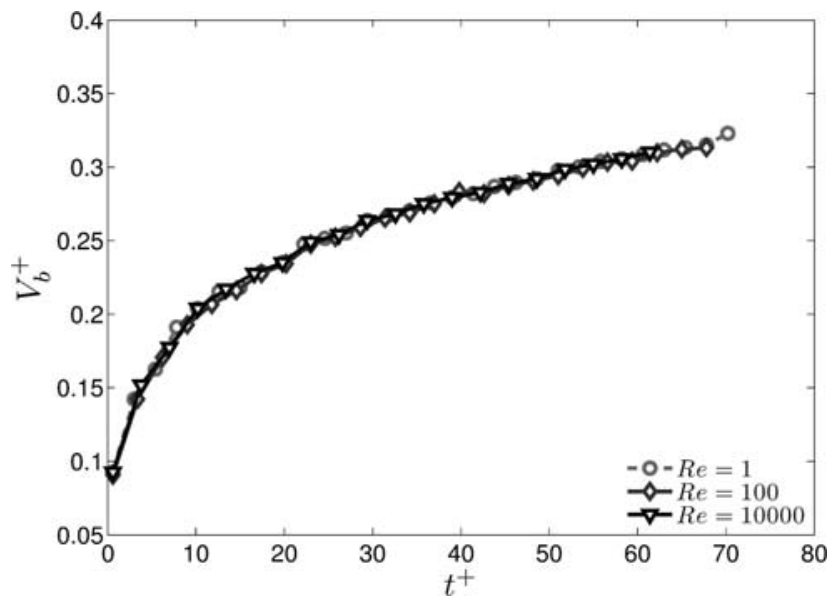

Figure 21. Reynolds number effect on liquid bridge filling, $h_{1}^{+}=0.04, h_{2}^{+}=0.08, S^{+}=0.02$.

[Color figure can be viewed in the online issue, which is available at wileyonlinelibrary.com.]
1. The time scale for bridge formation must be smaller 805 than a characteristic time scale (denoted as $t_{\text {acc }}$ ) for the par- 806 ticles to accelerate to a typical speed of liquid flow (i.e., 807 $\left.u_{\text {ref }}=\sigma / \mu_{l}\right)$. Physically this means that the speed of the parti- 808 cle relative motion is smaller than the speed of liquid flow. 809

2. The time scale for bridge formation must be smaller 810 than the time (denoted as $t_{\text {cross }}$ ) it takes for the particles to 811 cross the film.

The acceleration time scale $t_{\text {acc }}$ can be calculated from the 813 force balance on a particle. Assuming that the liquid bridge 814 only exhibits a cohesive force due to surface tension, the 815 dimensional acceleration time scale (such that the particle 816 have accelerated to the typical liquid flow speed $u_{\text {ref }}$ ) is 817 $t_{\text {acc }}=R^{2} \rho_{p} / \mu_{l}$. The corresponding dimensionless acceleration 818 time scale (with $t_{\text {ref }}=R \mu_{l} / \sigma$ being the reference time scale) is: 819

$$
t_{\mathrm{acc}}^{+}=\frac{R \rho_{p} \sigma}{\mu_{l}^{2}}
$$

The time for an approaching particle to cross the film, that 820 is, $t_{\text {cross }}$, can be calculated from a typical particle-particle rela- 821 tive velocity $u_{\text {rel }}$ and the film thickness, that is, $t_{\text {cross }}=h_{0} / u_{\text {rel }} .822$

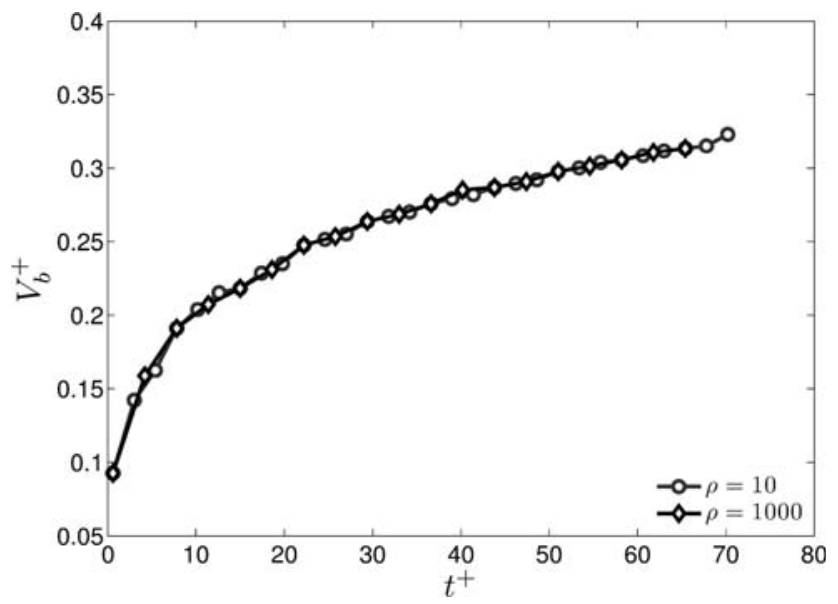

Figure 22. Density ratio effect on bridge volume filling, $h_{1}^{+}=0.04, h_{2}^{+}=0.08, S^{+}=0.02$.

[Color figure can be viewed in the online issue, which is available at wileyonlinelibrary.com.] 


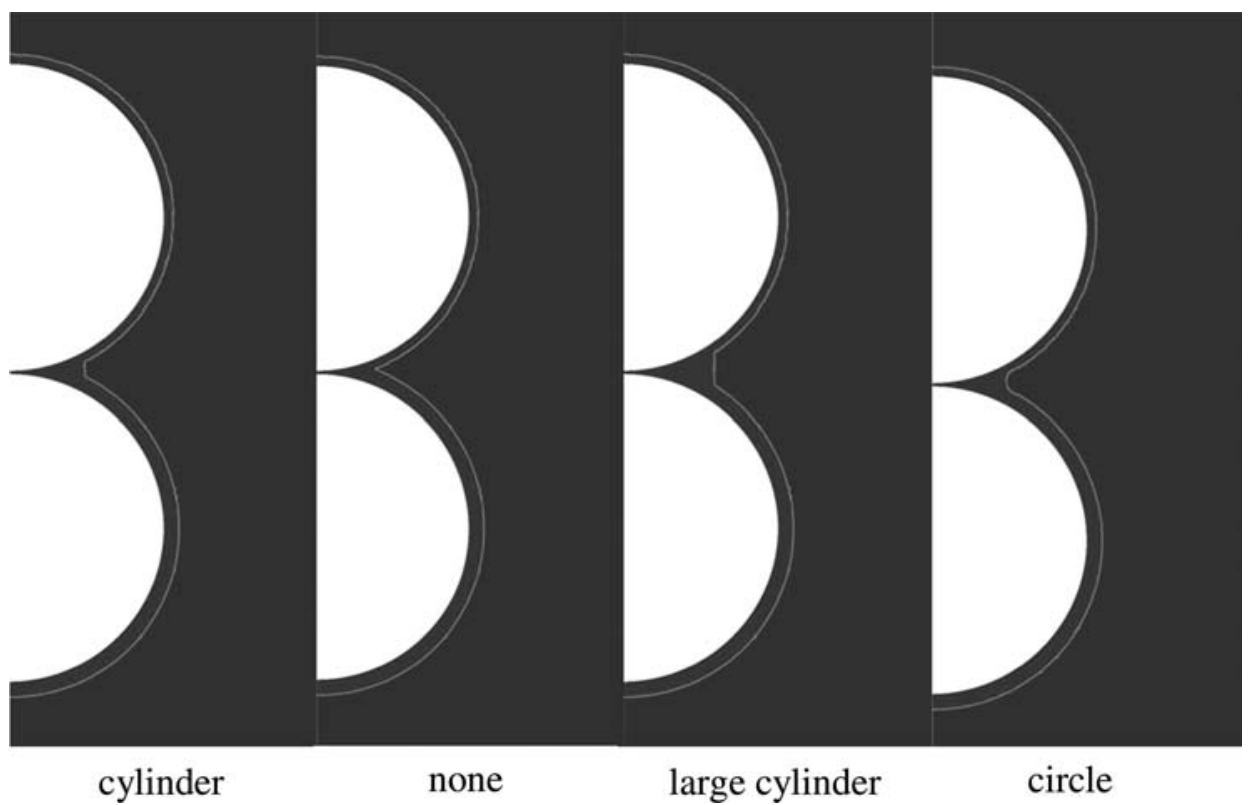

Figure 23. Different shapes of the initial liquid bridge $\left(h_{1}^{+}=0.06, h_{2}^{+}=0.10, S^{+}=0.01\right)$.

[Color figure can be viewed in the online issue, which is available at wileyonlinelibrary.com.]

Using Stokes settling velocity as $u_{\text {rel }}$, one obtains for the dimensionless crossing time scale:

$$
t_{\text {cross }}^{+}=\frac{9 h_{0}^{+} \mu_{g} \sigma}{2 R^{2} \mu_{l}\left(\rho_{p}-\rho_{g}\right) g}
$$

Here $h_{0}^{+}=h_{0} / R$ is the dimensionless film-thickness, $\mu_{g}$ is the ambient gas viscosity, and $\rho_{p}$ is the particle density. Note, that the alternative assumption of $u_{\mathrm{rel}}=u_{\mathrm{ref}}=\sigma / \mu$ would lead to $t_{\text {cross } u_{\text {ref }}}^{+}=h_{0}^{+}$. However, as explained above in the discussion of $t_{\text {acc }}$, it takes time to accelerate the particles to the capillary speed. Hence, the latter velocity scale is certainly of lower importance for typical applications that are characterized by a large particle Stokes number.

In case both dimensionless time scales are much larger than unity, the assumption of nonmoving particles in our simulations is acceptable. By assuming typical properties of various water-glycerine mixtures, ${ }^{49}$ and using parameters typical for a fluidized bed, we have summarized key dimensionless parameters in Table 2 and Figure 25. It can be seen that for most fluidized bed systems the assumption of zero relative particle velocity when modeling liquid transfer is justified. Tables 2 and 3 list relevant system parameters for particulate systems involving pure water, as well as mixtures of glycerine and water (60 and 40\%). In general, situations with highly viscous fluids (i.e., pure glycerine), appear to conflict with our quasistatic assumption. For these situations the relative velocity of the particles might influence the bridge filling process. However, for most systems involving typical liquids with a waterlike viscosity (see Tables 2 and 3, as well as the illustration in Figure 25), we find that the assumption of zero relative particle velocity is adequate.

\section{Viscous effects during particle approach}

The acceleration time scale defined above is based on Newton's law of motion considering capillary forces only. Clearly, viscous forces will retard the particles' relative motion, and hence we expect that the typical particle interaction time is larger than $t_{\mathrm{acc}}^{+}$. Next, we analyze such a situation by including lubrication effects due to the liquid between two approaching 857 wet particles. For such a situation we have:

$$
\vec{F}_{\text {vis }}+\vec{F}_{\text {cap }}=m \frac{d \vec{u}}{d t}
$$

where $\vec{F}_{\text {vis }}$ is the viscous force, $\vec{F}_{\text {cap }}$ is the capillary force, and $m$ is the mass of one particle. For small particles (i.e., in case 859 the particle size is smaller than the capillary length), the gravi- 860 tational forces can be neglected, and only capillary and vis- 861 cous forces affect particle motion. Pitois et al. ${ }^{14}$ and Darabi 862 et al. $^{2}$ applied the lubrication approximation for liquid flow 863 between the two particle surfaces, and arrived at the following 864 expression for the viscous force:

$$
\vec{F}_{\text {vis }}=-\frac{3}{2} \pi \mu R^{2} X_{v}^{2} \frac{1}{S} \frac{d S}{d t},
$$

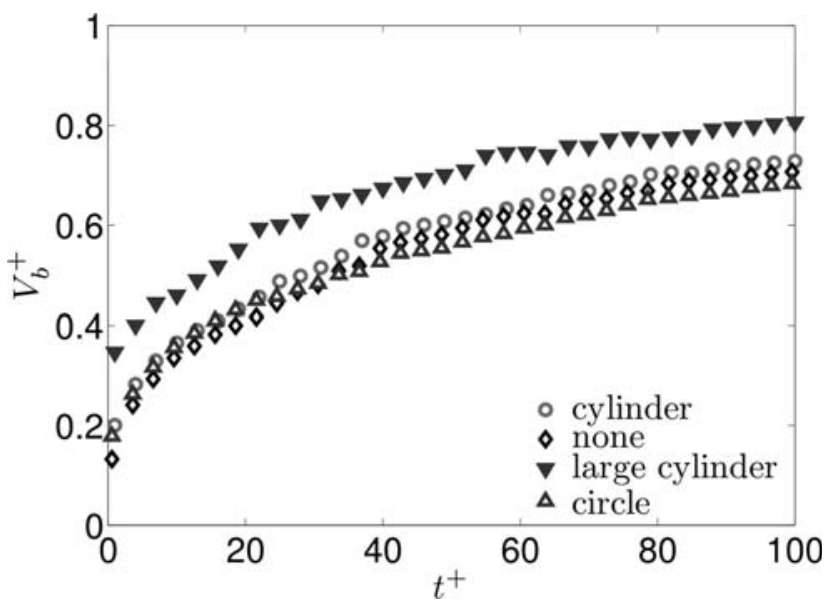

Figure 24. Effect of the initial bridge shape on the bridge filling process $\left(h_{1}^{+}=0.06, h_{2}^{+}=0.10\right.$, $S^{+}=0.01$ ).

[Color figure can be viewed in the online issue, which is available at wileyonlinelibrary.com.] 
Table 2. Summary of Parameters Relevant for Liquid Transfer in Typical Fluidized Beds (for $t_{\text {cross }}^{+}$Refer to Figure 25)

\begin{tabular}{|c|c|c|c|c|c|c|}
\hline Glycerine/water & $R[\mathrm{~m}]$ & $\rho_{p}\left[\mathrm{~kg} \cdot \mathrm{m}^{-3}\right]$ & $h_{0}^{+}$ & $t_{\mathrm{ref}}[\mathrm{s}]$ & $t_{\mathrm{acc}}^{+}$ & $O h$ \\
\hline Water & $5 e-6$ & 1000 & 0.01 & $6.86 e-8$ & 363.5 & 0.052 \\
\hline Glycerine/water-60/40\% & $5 e-6$ & 1000 & 0.01 & $8.54 \mathrm{e}-7$ & 2.54 & 0.58 \\
\hline Glycerine/water-79/21\% & $5 e-6$ & 1000 & 0.01 & $3.86 e-6$ & 0.13 & 2.53 \\
\hline Glycerine/water- $90 / 10 \%$ & $5 e-6$ & 1000 & 0.01 & $1.74 \mathrm{e}-5$ & $6.55 e-3$ & 11.1 \\
\hline Pure glycerine & $5 e-6$ & 1000 & 0.01 & $8.87 e-5$ & $2.52 \mathrm{e}-4$ & 56.13 \\
\hline Water & $5 e-6$ & 1000 & 0.1 & $6.86 \mathrm{e}-8$ & 363.5 & 0.052 \\
\hline Glycerine/water-60/40\% & $5 e-6$ & 1000 & 0.1 & $8.54 \mathrm{e}-7$ & 2.54 & 0.58 \\
\hline Glycerine/water-79/21\% & $5 e-6$ & 1000 & 0.1 & $3.86 \mathrm{e}-6$ & 0.13 & 2.53 \\
\hline Glycerine/water-90/10\% & $5 e-6$ & 1000 & 0.1 & $1.74 \mathrm{e}-5$ & $6.55 e-3$ & 11.1 \\
\hline Pure glycerine & $5 e-6$ & 1000 & 0.1 & $8.87 \mathrm{e}-5$ & $2.52 \mathrm{e}-4$ & 56.13 \\
\hline Water & $5 e-3$ & 1000 & 0.1 & $6.86 e-5$ & $3.64 \mathrm{e} 5$ & $1.66 \mathrm{e}-3$ \\
\hline Glycerine/water-60/40\% & $5 e-3$ & 1000 & 0.1 & $8.54 \mathrm{e}-4$ & $2.54 \mathrm{e} 3$ & $1.85 \mathrm{e}-2$ \\
\hline Glycerine/water-79/21\% & $5 e-3$ & 1000 & 0.1 & $3.86 e-3$ & 130 & 0.08 \\
\hline Glycerine/water- $90 / 10 \%$ & $5 e-3$ & 1000 & 0.1 & $1.74 \mathrm{e}-2$ & 6.55 & 0.35 \\
\hline Pure glycerine & $5 e-3$ & 1000 & $1 e-4$ & $8.87 \mathrm{e}-2$ & 0.25 & 1.77 \\
\hline Water & $5 e-6$ & 5000 & 0.1 & $6.86 \mathrm{e}-8$ & 1717 & 0.052 \\
\hline Glycerine/water-60/40\% & $5 e-6$ & 5000 & 0.1 & $8.54 \mathrm{e}-7$ & 12.7 & 0.58 \\
\hline Glycerine/water-79/21\% & $5 e-6$ & 5000 & 0.1 & $3.86 \mathrm{e}-6$ & 0.65 & 2.53 \\
\hline Glycerine/water-90/10\% & $5 e-6$ & 5000 & 0.1 & $1.74 \mathrm{e}-5$ & 0.033 & 11.1 \\
\hline Pure glycerine & $5 e-6$ & 5000 & 0.1 & $8.87 \mathrm{e}-5$ & $1.25 \mathrm{e}-3$ & 56.13 \\
\hline
\end{tabular}

$$
X_{v}=1-1 / \sqrt{1+\frac{2 V_{b}}{\pi R S^{2}}} .
$$

where $R$ is the particle radius, $S$ is the half separation distance between the particle surfaces, and $V_{b}$ is the liquid bridge volume. Pitois et al. ${ }^{14}$ and Darabi et al. ${ }^{2}$, also provided a model for the capillary force that accounts for the bridge volume effect. For fully wetted particles their capillary force model is:

$$
\vec{F}_{\text {cap }}=2 \pi R \sigma X_{v}
$$

Substituting the model for the viscous and capillary force into Eq. 25, and using typical initial conditions (i.e., an initial separation of $10 \%$ of the particle radius, and particles initially at rest), we obain the following differential equation:

$$
\left\{\begin{array}{l}
-\frac{3}{2} \pi \mu_{l} R^{2} X_{v}^{2} \frac{1}{S} \frac{d S}{d t}+2 \pi R \sigma X_{v}=m \frac{d^{2} S}{d t^{2}} \\
S(0)=0.1 R ; \quad S^{\prime}(0)=0 .
\end{array}\right.
$$

Integrating the above equation in time is straight forward (e.g., using Matlab ${ }^{\circledR}$ ), and we have chosen two sets of parame-

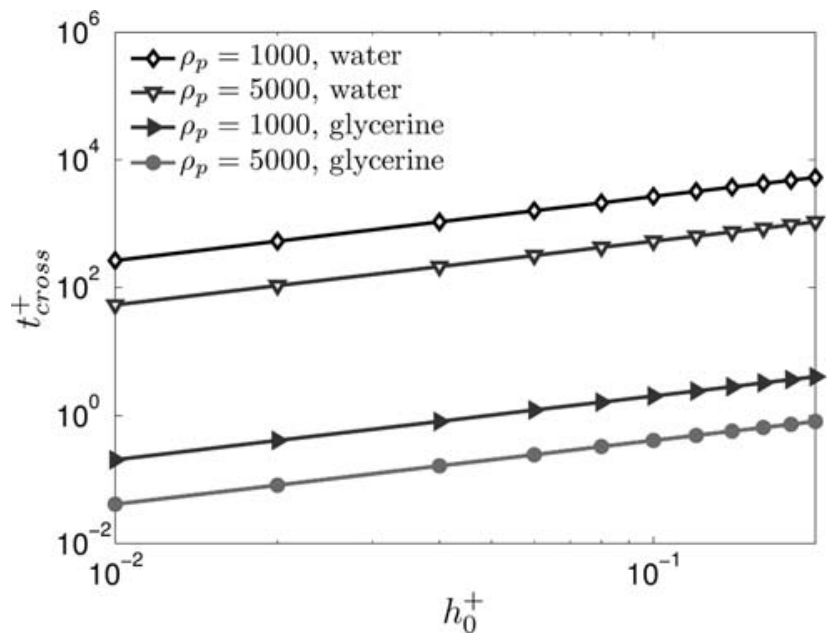

Figure 25. $t_{\text {cross }}^{+}$for water and pure glycerine in typical fluidized bed operations.

[Color figure can be viewed in the online issue, which is available at wileyonlinelibrary.com.] ters, that is, that of water and pure glycerine to illustrate the 877 solution. Relevant properties for these liquids are listed in 878 Table 4. The particle size is chosen to be $10 \mu \mathrm{m}$, the liquid $879 \mathrm{~T} 4$ bridge volume is chosen to be $20 \%$ of the particle volume, the 880 Reynold number is defined by the capillary speed, the particle 881 radius, and the fluid viscosity (i.e., $\mathrm{Re}=\sigma R \rho_{l} / \mu_{l}^{2}$ ). One can 882 also define a capillary number $C a$ to quantify the ratio of vis- 883 cous and capillary effects. This number is based on the Stokes 884 setting velocity (as a proxy for the relevant particle-particle 885 relative speed), the viscosity of the liquid, and the surface ten- 886 sion, that is, $C a=\mu_{l} u_{\text {rel }} / \sigma=\left(2 \mu_{l} R^{2}\left(\rho_{p}-\rho_{g}\right) g\right) /\left(9 \mu_{g} \sigma\right)$. In 887 summary, we obtain for - pure glycerine: $R e=6.34 \times 10^{-4}, \mathrm{Ca}=0.975$, and for 889 - water: $\operatorname{Re}=730, \mathrm{Ca}=7.54 \times 10^{-4}$

Figure 26 illustrates the time evolution of the particle sepa- 89 F26 ration distance, as well as the acceleration time scale derived 892 above. Figure 27 summarizes the corresponding relative veloc- 89 F27 ity scaled with the reference velocity, that is, a typical speed 894 of the adhering liquid when flowing into the bridge. We can 895 observe from Figure 26 (dashed and continuous bold line) that 896 the time until contact is larger than $t_{\text {acc }}^{+}$for the glycerine 897

Table 3. Effect of Selected Particle Parameters on Dimensionless Bridge Filling Parameters for Glycerine/ Water-60/40\% Mixtures

\begin{tabular}{lccccccc}
\hline Cases & $R[\mathrm{~m}]$ & $\rho_{p}\left[\mathrm{~kg} \cdot \mathrm{m}^{-3}\right]$ & $h_{0}^{+}$ & $t_{\text {ref }}[\mathrm{s}]$ & $t_{\text {acc }}^{+}$ & $t_{\text {cross }}^{+}$ & $O h$ \\
\hline 1 & $5 \mathrm{e}-6$ & 1500 & $1 \mathrm{e}-2$ & $8.54 \mathrm{e}-7$ & 3.82 & 14.2 & 0.58 \\
2 & $5 \mathrm{e}-6$ & 1500 & 0.2 & $8.54 \mathrm{e}-7$ & 3.82 & 284 & 0.58 \\
3 & $1 \mathrm{e}-5$ & 1500 & 0.1 & $1.71 \mathrm{e}-6$ & 7.63 & 35.5 & 0.41 \\
4 & $1 \mathrm{e}-5$ & 2000 & 0.1 & $1.71 \mathrm{e}-6$ & 10.18 & 26.6 & 0.41 \\
5 & $1 \mathrm{e}-5$ & 5000 & 0.1 & $1.71 \mathrm{e}-6$ & 25.44 & 10.6 & 0.41 \\
\hline
\end{tabular}

Table 4. Properties of Different Water-Glycerine Mixtures (Adapted from Eddi et al. ${ }^{49}$ )

\begin{tabular}{lccl}
\hline Mixture & $\mu[\mathrm{Pa} \cdot \mathrm{s}]$ & $\rho\left[\mathrm{kg} \cdot \mathrm{m}^{-3}\right]$ & $\sigma[\mathrm{N} / \mathrm{m}]$ \\
\hline Water & $1 \times 10^{-3}$ & 1000 & 0.073 \\
Glycerine/water-60/40\% & 0.0115 & 1153 & 0.0673 \\
Glycerine/water-79/21\% & 0.05 & 1204 & 0.0647 \\
Glycerine/water-90/10\% & 0.22 & 1238 & 0.0634 \\
Pure glycerine & 1.12 & 1262 & 0.0631 \\
\hline
\end{tabular}




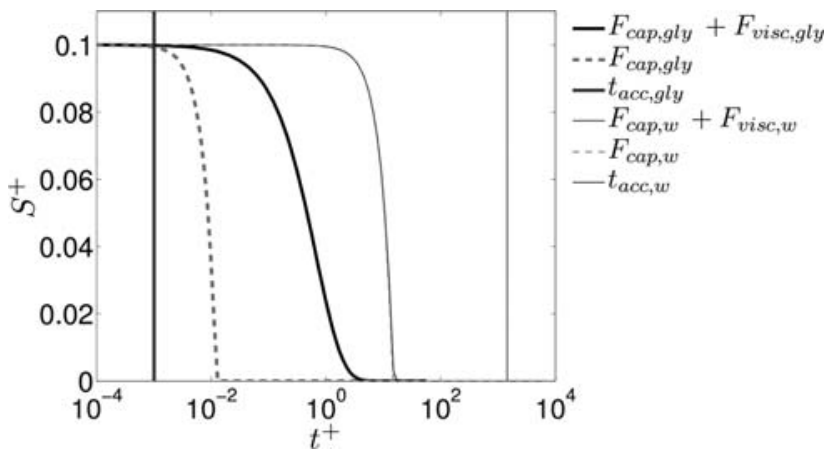

Figure 26. Time evolution of the half separation distance $S^{+}$during the acceleration phase of two approaching particles $\left(\rho_{p}=2000[\mu \mathrm{m}]\right.$, $d_{p}=10[\mu \mathrm{m}], V_{b}^{+}=0.1$ ).

[Color figure can be viewed in the online issue, which is available at wileyonlinelibrary.com.]

system. Thus, the particles accelerate, but only for the situation where viscous forces are neglected the particle accelerate beyond $u_{\text {ref }}$ (see Figure 27). In contrast, in the system involving water the particles' relative speed never excees $u_{\text {ref }}$, and the impact speed is in the order of $0.01 u_{\text {ref }}$ (see thin dashed and continuous line in Figures 26 and 27). As expected, lubrication forces resist the particle's relative motion and delay the time until contact (see continuous lines in Figures 26 and 27). This effect is more pronounced for the viscous systems, while the effect in the system involving water is rather small. Clearly, in case one does not take into account the viscous term (see Eq. 26) for the glycerine system, the particles accelerate to an unphysically large relative velocity. In case we account for viscous forces, however, we expect typical particle relative speeds smaller than $0.1 u_{r e f}$, even for highly viscous systems (see Figure 27). These particle velocities are below the typical liquid speed for early times (i.e., $t^{+}=1$, see Figure 2). For longer times both the liquid and particle speed decreases rapidly, the particles' surfaces touch (at $t^{+}$between 5 and 50 depending on the viscosity of the liquid, see

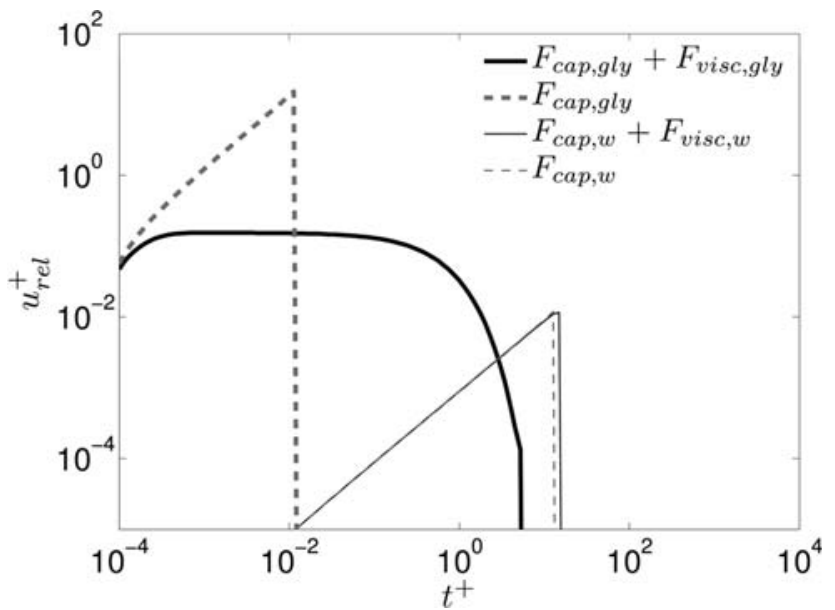

Figure 27. Time evolution of the relative particle velocity during the acceleration phase of two approaching particles (parameters are the same as in Figure 26).

[Color figure can be viewed in the online issue, which is available at wileyonlinelibrary.com.]

Figure 27). Hence, bridge filling is also unaffected by particle 918 motion for longer times, simply because the particles are 919 already in contact. Based on the above consideration of par- 920 ticles accelerating from zero velocity, it appears that our 921 assumption of a quasi-static liquid bridge formation is valid 922 for a wide range of wet particulate systems.

\section{Typical impact speeds in sedimenting suspensions}

The typical relative particle velocity at impact is important 925 for our analysis, since it affects the scale for film crossing. We 926 have therefore performed simulations of a typical application 927 we are interested in (i.e., wet granulation in a fluidized bed). 928 Specifically, we considered a freely sedimenting suspension, 929 and have recorded the speed and orientation of particle-parti- 930 cle collisions. The simulations followed the approach used by 931 Radl and Sundaresan ${ }^{50}$, with identical fluid and particle 932

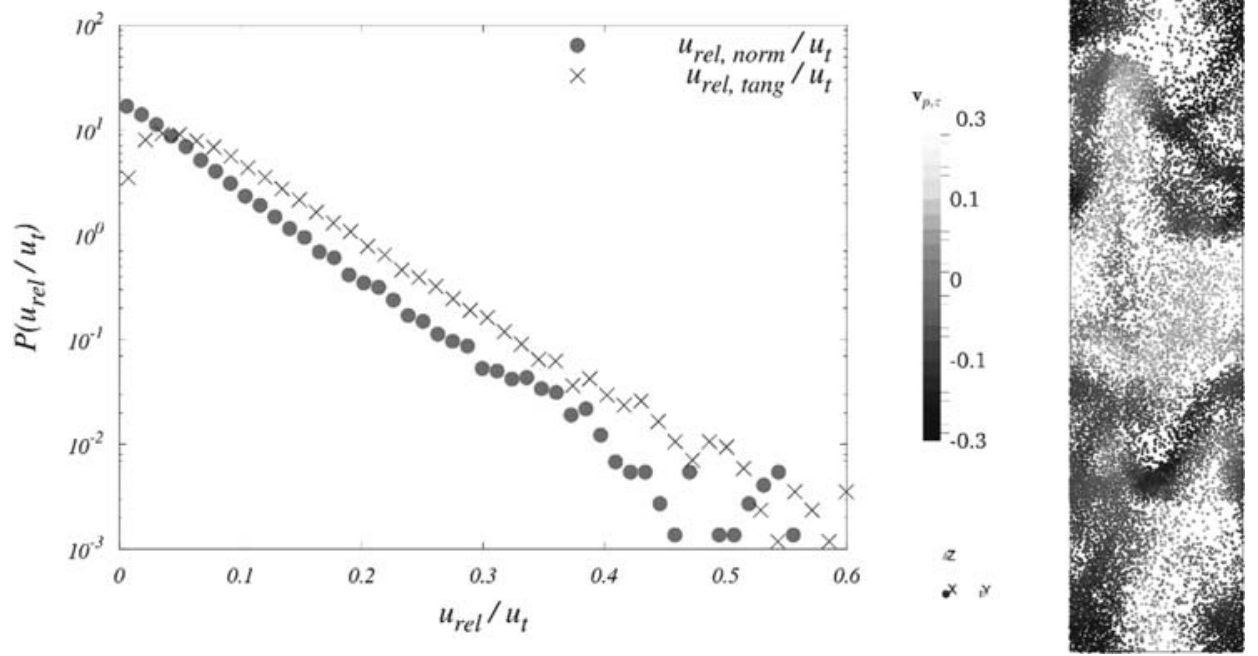

Figure 28. Distribution of particle collision velocities in the normal and tangential direction (left panel), as well as illustration of the vertical particle velocity distribution (right panel, $d_{p}=150[\mu \mathrm{m}], \rho_{p}=1500\left[\mathrm{~kg} / \mathrm{m}^{3}\right]$, $\phi_{p}=0.10$, particles sediment in air at ambient conditions).

[Color figure can be viewed in the online issue, which is available at wileyonlinelibrary.com.] 
properties. Our results are summarized in Figure 28, highlighting that collisions in typical applications we are interested in (i.e., fluidized beds) are mostly gentle: the typical impact speed is in the order of $10 \%$ of the particles' terminal settling velocity. Also, we observe from Figure 28 that particle collisions are primarily oblique, that is, the particles' relative speed in the tangential direction (at the contact point) is smaller than that in the normal direction. In Appendix $\mathrm{C}$ we summarize more data for sedimenting suspensions, which show a similar qualitative behavior and a moderate increase of the impact speed when decreasing the particle concentration. Clearly, our data supports our assumption of quasi-static bridge filling for a wide range of wet particulate systems with rather thick liquid films and a rather low liquid viscosity.

Of course, the relative speed of particles in a wet collision event is set by the process, and we have only considered a wet fluidized bed here. Clearly, it is necessary to check the appropriateness of our model for a specific application via a prior analysis of typical particle relative velocities.

\section{Conclusions}

A new model to predict dynamic liquid bridge formation between two wet particles has been presented in this article. This model is based on DNS data, which were obtained by extracting the interface position from VoF-based simulations of the bridge filling process. The liquid bridge volume was defined based on a characteristic neck position, and a DIM was employed to calculate the liquid bridge volume. This allowed us building a dynamic model for predicting the bridge volume, and the liquid remaining on the particle surfaces. Such a model might help to refine our picture of wet particle collisions that previously focused exclusively on predictions of the coefficient of restitution (Donahue et al. ${ }^{24}$, Sutkar et al. $\left.^{28}\right)$.

Our model differentiates between (1) a fast initial bridge formation stage where the dimensionless time is less than a reference time for capillary-driven viscous flow, and (2) a subsequent slower viscous filling stage where viscous effects are dominant. The initial stage model is based on a geometrical reference volume, and has been calibrated with DNS data at a dimensionless time of $t^{+}=1$. Our initial stage model can be used as a first estimate for the liquid bridge volume in short particle collisions, and is an extension of the model prosed by Shi and McCarthy ${ }^{46}$. The postulated model for the viscous filling stage model relies on a universal parameter $a_{i}$ (i.e., a characteristic dimensionless filling time), as well as dimensionless liquid mobility parameters $\phi_{m 1}$ and $\phi_{m 2}$ of the contacting particles. A model equation for these mobility parameters has been proposed. Specifically, we consider that the mobilities are functions of the film height and the separation distance. In summary, our model is valid for liquid bridge formation between two identical particles coated with thin continuous films (i.e., an initial relative film height of less than $10 \%$ of the particle radius).

We observed that our results obtained from the DNS are independent of the Reynolds number, as well as the density ratio between the liquid coating the particles and the ambient gas. This suggests that our model is applicable to a wide range of gas-particle systems involving wet particulate systems.

However, our simulation study indicates that grid refinement plays an important role in the final stages of film flow where the film ruptures. To get a precise model for the filling process at long times, as well as to correctly predict film rup- ture, it is essential to use a fine enough computational mesh in 995 the simulations (i.e., the dimensionless grid resolution $\Delta h 996$ should be 0.12 or smaller). This clearly limited the current 997 study to axisymmetric configurations. Hence, our study is only 998 a step forward to better understand the equilibration of liquid 999 on particles and in liquid bridged in a particle bed. Still work 1000 needs to be done in the future, specifically, it would be inter- 1001 esting to

- experimentally support the observed film rupturing event 1003 for long times,

- investigate the wetting of initially completely dry particle, 1005 and particles that have a complex morphology, 1006 - quantify the effect of particle relative motion on the liquid 1007 bridge formation process.

\section{Acknowledgments}

We acknowledge the funding of the FWF through project 1010 P23617, and we gratefully acknowledge support from NAWI 1011 Graz by providing access to dcluster.tugraz.at. The authors 1012 would like to thank Ben Freireich (DOW Chemicals) for 1013 fruitful discussions on time scales associated with wet parti- 1014 cle collisions, and the anonymous reviewers for their valua- 1015 ble comments.

\section{Notation}

\section{Latin symbols}

$a_{i}=$ dimensionless filling rate parameter, -

$\mathrm{Ca}=$ capillary number, -

$d_{p}=$ particle diameter, $\mathrm{m}$

$\vec{F}_{\text {vis }}=$ viscous force acting on the particle, $\mathrm{kg} \cdot \mathrm{m} / \mathrm{s}^{2}$

$\vec{F}_{\text {cap }}=$ capillary force acting on the particle, $\mathrm{kg} \cdot \mathrm{m} / \mathrm{s}^{2}$

$g=$ gravity, $\mathrm{m} / \mathrm{s}^{2}$

$h_{0}=$ average initial film height of the particle pair, $\mathrm{m}$

$h_{i}=$ initial film height of particle $i, \mathrm{~m}$

$h_{\mathrm{cyl}, i}=$ the height of the red shade cylinder on particle $i, \mathrm{~m}$

$\mathrm{L}_{p, 0}=$ reference volume of liquid on the particle, $\mathrm{m}^{3}$

$\mathrm{L}_{p, i}=$ volume of liquid present on the particle $i, \mathrm{~m}^{3}$

$m=$ mass of the particle, $\mathrm{kg}$

$\mathbf{n}_{\mathrm{ij}}=$ unit normal vector, -

$O h=$ Ohnesorge number, -

$P=$ pressure, $\mathrm{Pa}$

$P_{\text {ref }}=$ reference pressure, $\mathrm{Pa}$

$p_{s}=$ pressure at the particle surfaces, $\mathrm{Pa}$

$p_{V_{b}}=$ pressure at the liquid bridge, $\mathrm{Pa}$

$R=$ particle radius, $\mathrm{m}$

$R_{\text {cyl }}=$ radius of the initial cylinder region, $\mathrm{m}$

$R_{\text {curve }}=$ radius of curvature of the liquid bridge surface, $\mathrm{m}$

$R e=$ reynolds number, -

$S=$ half separation distance between particles, $\mathrm{m}$

$t=$ time, $\mathrm{s}$

$t_{\text {acc }}=$ acceleration time scale, $\mathrm{s}$

$t_{\text {cross }}=$ film crossing time scale, $\mathrm{s}$

$t_{\text {relax }}=$ particle relation time, $\mathrm{s}$

$t_{\text {ref }}=$ reference time scale, $\mathrm{s}$

$u_{\text {ref }}=$ reference fluid velocity, $\mathrm{m} \cdot \mathrm{s}$

$u_{\mathrm{rel}}=$ relative particle-particle velocity, $\mathrm{m} / \mathrm{s}$

$U=$ fluid velocity, $\mathrm{m} / \mathrm{s}$

$V_{b}=$ liquid bridge volume, $\mathrm{m}^{3}$

$V_{b, 0}=$ initial bridge volume, $\mathrm{m}^{3}$

$\begin{aligned} V_{b, O, I} & =\text { geometry bridge volume: type } \mathrm{I}, \mathrm{m}^{3}\end{aligned}$

$V_{b, g, I I}=$ geometry bridge volume: type II, $\mathrm{m}^{3}$

$V_{b, i, I}=$ integration volume of the red framed region in Figure $4 \mathrm{~b}, \mathrm{~m}^{3} 112 \not b$

$V_{\text {cap }, i}=$ the cavity volume of particle, $\mathrm{m}^{3}$

$V_{\text {overLap }}=$ the displaced volume of liquid of the overlap region, $\mathrm{m}^{3}$

$\mathrm{DIM}=$ direct integration method

$\mathrm{DNS}=$ direct numerical simulation

$\mathrm{MFB}=$ micro force balance

YLE $=$ Young-Laplace equation 


\section{Greek symbols}

$\alpha=$ phase fraction indicator, -

$\beta_{\mathrm{cyl}, i}=$ initial filling angle on particle $i$ that cause by geometry bride, $\mathrm{rad}$

$\Delta t=$ time step, $\mathrm{s}$

$\Delta x=$ grid spacing, $\mathrm{m}$

$\Delta h=$ dimensionless grid spacing by initial film height, -

$\phi_{p}=$ particle volume fraction, -

$\phi_{m i}=$ fraction of liquid on particle $i$ that is mobile to flow into the bridge, -

$\mu_{l}=$ dynamic viscosity of liquid, $\mathrm{kg} \mathrm{m}^{-1} \mathrm{~s}^{-1}$

$\mu_{g}=$ dynamic viscosity of ambient gas, $\mathrm{kg} \mathrm{m}^{-1} \mathrm{~s}^{-1}$

$\rho_{l}=$ density of the liquid, $\mathrm{kg} \mathrm{m}^{-3}$

$\rho_{g}=$ density of the ambient gas, $\mathrm{kg} \mathrm{m}^{-3}$

$\rho_{p}=$ density of the particles, $\mathrm{kg} \mathrm{m}^{-3}$

$\sigma=$ surface tension, $\mathrm{kg} \mathrm{s}^{-2}$

\section{Superscripts}

$+=$ dimensionless quantity

$i=$ particle index

norm $=$ normal direction

$\operatorname{tang}=$ tangential direction

$t=$ terminal

$w=$ water

gly $=$ glycerine

$P=$ particle

ref $=$ reference quantity

\section{Literature Cited}

1. Toschkoff G, Khinast JG. Mathematical modeling of the coating process. Int J Pharm. 2013;457(2):407-422. doi:10.1016/j.ijpharm.2013.08.022.

2. Darabi P, Pougatch K, Salcudean M, Grecov D. A novel coalescence model for binary collision of identical wet particles. Chem Eng Sci. 2009;64:1868-1876.

3. Scheel M, Seemann R, Brinkmann M, Di Michiel M, Sheppard A, Breidenbach B, Herminghaus S. Morphological clues to wet granular pile stability. Nat Mater. 2008;7:189-193. doi:10.1038/nmat2117.

4. Orr FM, Scriven LE, Rivas AP. Pendular rings between solids: meniscus properties and capillary force. J Fluid Mech. 1975;67(04): 723. doi: $10.1017 / \mathrm{S} 0022112075000572$

5. Darabi P, Li T, Pougatch K, Salcudean M, Grecov D. Modeling the evolution and rupture of stretching pendular liquid bridges. Chem Eng Sci. 2010;65:4472-4483. doi:10.1016/j.ces.2010.04.003.

6. Dodds S, Carvalho M, Kumar S. Stretching liquid bridges with moving contact lines: the role of inertia. Phys Fluids. 2011;23. doi: $10.1063 / 1.3623427$.

7. Dodds S, Carvalho MS, Kumar S. The dynamics of threedimensional liquid bridges with pinned and moving contact lines. J Fluid Mech. 2012;707:521-540. doi:10.1017/jfm.2012.296.

8. Pepin X, Rossetti D, Iveson S, Simons S. Modeling the evolution and rupture of pendular liquid bridges in the presence of large wetting hysteresis. J Colloid Interface Sci. 2000;232(2):289-297. doi: 10.1006/jcis.2000.7182.

9. Rossetti D, Simons SJR. A microscale investigation of liquid bridges in the spherical agglomeration process. Powder Technol. 2003; 130(1-3):49-55. doi:10.1016/S0032-5910(02)00225-5.

10. Herminghaus S. Dynamics of wet granular matter. Adv Phys. 2005; 54(3):221-261. doi:10.1080/00018730500167855.

11. Haines WB. Studies in the physical properties of soils: II. A note on the cohesion developed by capillary forces in an ideal soill. J Agric Sci. 1925;15:529-535. doi:http://dx.doi.org/10.1017/S0021859600082460.

12. Fisher R. A. On the capillary forces in an ideal soil: correction of formulae given by WB haines. J Agric Sci. 1926;16:492-505. doi: http://dx.doi.org/10.1017/S0021859600007838.

13. Mehrotra VP, Sastry KVS. Pendular bond strength between unequalsized spherical particles. Powder Technol. 1980;25:203-214.

14. Pitois O, Moucheront P, Chateau X. Liquid bridge between two moving spheres: an experimental study of viscosity effects. $J$ Colloid Interface Sci. 2000;231:26-31. doi:10.1006/jcis.2000.7096.

15. McLaughlin LJ, Rhodes MJ. Prediction of fluidized bed behaviour in the presence of liquid bridges. Powder Technol. 2001;114:213-223. doi:10.1016/S0032-5910(00)00325-9.
16. Grof Z, Lawrence CJ, Stepánek F. The strength of liquid bridges in 1262 random granular materials. J Colloid Interface Sci. 2008;319(1):182- 1263 192. doi:10.1016/j.jcis.2007.11.055.

17. Rabinovich YI, Esayanur MS, Moudgil BM. Capillary forces between 1265 two spheres with a fixed volume liquid bridge: theory and experiment. 1266 Langmuir. 2005;21:10992-10997. doi:10.1021/la0517639. 1267

18. Wei Z, Zhao Y-P. Growth of liquid bridge in AFM. J Phys D Appl 1268 Phys. 2007;40:4368-4375. doi:10.1088/0022-3727/40/14/036. 1269

19. Muguruma Y, Tanaka T, Tsuji Y. Numerical simulation of particu- 1270 late flow with liquid bridge between particles (simulation of centrifu- 1271 gal tumbling granulator). Powder Technol. 2000;109:49-57. doi: 1272 10.1016/S0032-5910(99)00226-0.

20. Shi D, Vargas WL, McCarthy JJ. Heat transfer in rotary kilns with 1274 interstitial gases. Chem Eng Sci. 2008;63(18):4506-4516. doi: 1275 10.1016/j.ces.2008.06.006.

21. De Bisschop FR., Rigole WJ. A physical model for liquid capillary 1277 bridges between adsorptive solid spheres: the nodoid of plateau. 1278 $J$ Colloid Interface Sci. 1982;88:117-128. doi:10.1016/0021-1279 9797(82)90161-8.

22. Chen Y, Zhao Y, Gao H, Zheng J. Liquid bridge force between two 1281 unequal-sized spheres or a sphere and a plane. Particuology. 2011;9: 1282 374-380. doi:10.1016/j.partic.2010.11.006.

23. Davis RH, Rager DA, Good BT. Elastohydrodynamic rebound of 1284 spheres from coated surfaces. J Fluid Mech. 2002;468:107-119. doi: 1285 $10.1017 / \mathrm{S} 0022112002001489$

24. Donahue CM, Hrenya CM, Davis RH. Stokes's cradle: Newton's 1287 cradle with liquid coating. Phys Rev Lett. 2010;105(3). doi:10.1103/1288 PhysRevLett.105.034501.

25. Li X, Hunt ML, Colonius T. A contact model for normal immersed 1290 collisions between a particle and a wall. J Fluid Mech. 2012;691: 1291 123-145. doi:10.1017/jfm.2011.461.

26. Gondret P, Lance M, Petit L. Bouncing motion of spherical particles 1293 in fluids. Phys Fluids. 2002;14(2):643-652. doi:10.1063/1.1427920. 1294

27. Gollwitzer F, Rehberg I, Kruelle CA, Huang K. Coefficient of resti- 1295 tution for wet particles. Phys Rev E Stat Nonlinear, Soft Matter 1296 Phys. 2012;86(1):1-10. doi:10.1103/PhysRevE.86.011303. 1297

28. Sutkar VS, Deen NG, Johan TP, Kuiper JAM, Salikov V, Cruger B, 1298 Antonyuk S, Heinrich S. A novel approach to determine wet restitu- 1299 tion coefficients through a unified correlation and energy analysis. 1300 AIChE J. 2015;61:769-779. doi:10.1002/aic. 1301

29. Kantak AA, Hrenya MC, Davis HR. Initial rates of aggregation for 1302 dilute, granular flows of wet particles. Phys Fluids. 2009;21(2). doi: 1303 10.1063/1.3070830.

30. Yang F. Interaction law for a collision between two solid particles in 1305 a viscous liquid. 2006;2006.

31. Štěpánek F, Ansari MA. Computer simulation of granule microstruc- 1307 ture formation. Chem Eng Sci. 2005;60:4019-4029. doi:10.1016/1308 j.ces.2005.02.030.

32. Lian G, Thornton C, Adams MJ. A theoretical study of the liquid 1310 bridge forces between two rigid spherical bodies. J Colloid Interface 1311 Sci. 1993;161:138-147. doi:http://dx.doi.org/10.1006/jcis.1993.1452. 1312

33. Erle MA, Dyson DC, Morrow NR. Liquid bridges between cylinders, 1313 in a torus, and between spheres. AIChE J. 1971;17:115-121. doi: 1314 10.1002/aic.690170125.

34. Mikami T, Kamiya H, Horio M. Numerical simulation of cohesive 1316 powder behavior in a fluidized bed. Chem Eng Sci. 1998;53:1927- 1317 1940. doi:10.1016/S0009-2509(97)00325-4.

35. Willett CD, Adams MJ, Johnson SA, Seville JPK. Capillary bridges 1319 between two spherical bodies. Langmuir. 2000;16:9396-9405. doi: 1320 10.1021/la000657y.

36. Harireche O, Faramarzi A, Alani AM. A toroidal approximation of 1322 capillary forces in polydisperse granular assemblies. Granul Matter. 1323 2013;15(5):573-581. doi:10.1007/s10035-013-0425-9. 1324

37. Simons SJR, Seville JPK, Adams MJ. An analysis of the rupture 1325 energy of pendular liquid bridges. Chem Eng Sci. 1994;49(14):2331- 1326 2339. doi:10.1016/0009-2509(94)E0050-Z.

38. Mazzone DN, Tardos GI, Pfeffer, R. The behavior of liquid bridge 1328 between two relatively moving particles. Powder Technol. 1987;51: 1329 $71-83$.

39. Eggers J, Dupont TF. Drop formation in a one-dimensional approxi- 1331 mation of the Navier-Stokes equation. J Fluid Mech. 1994;262:205- 1332 221. doi: $10.1017 / \mathrm{S} 0022112094000480$.

40. Papageorgiou DT. On the breakup of viscous liquid threads. Phys 1334 Fluids. 1995;7(7):1529. doi:10.1063/1.868540.

41. Zhang X, Padgett RS, Basaran OA. Nonlinear deformation and 1336 breakup of stretching liquid bridges. J Fluid Mech. 1996;329:207. 1337 doi:10.1017/S0022112096008907. 
42. Vega EJ, Montanero JM, Herrada MA., Ferrera C. Dynamics of an axisymmetric liquid bridge close to the minimum-volume stability limit. Phys Rev E. 2014;90(1):013015. doi:10.1103/ PhysRevE.90.013015.

43. Wu M, Khinast J, Radl S. Direct simulation of film flow on spheres to investigate liquid bridge. In: 2014 AICHE Annual Meeting, November 16. Atlanta, Vereinigte Staaten (USA); 2014.

44. Easo LA, Kumar R, Ren R, Carl R. Numerical study of the formation of a liquid bridge between two spheres with uniform film thickness. In: 2014 AICHE Annual Meeting, November 18. 2014.

45. Radl S, Khinast JG, Sundaresan S. On the filling rate of a liquid bridge between wet particles. In: 2013 AICHE Annual Meeting, November 03-08. San Francisco, Vereinigte Staaten (USA); 2013.

46. Shi D, McCarthy JJ. Numerical simulation of liquid transfer between particles. Powder Technol. 2008;184:64-75. doi:10.1016/ j.powtec.2007.08.011.

47. Mohan B, Kloss C, Khinast J, Radl S. Regimes of liquid transport through sheared beds of inertial smooth particles. Powder Technol. 2014;264:377-395. doi:10.1016/j.powtec.2014.05.045.

48. Deshpande SS, Anumolu L, Trujillo MF. Evaluating the performance of the two-phase flow solver interFoam. Comput Sci Discov. 2012; 5(1):014016. doi:10.1088/1749-4699/5/1/014016.

49. Eddi a., Winkels KG, Snoeijer JH. Short time dynamics of viscous drop spreading. Phys Fluids. 2013;25(1):013102. doi:10.1063/ 1.4788693 .

50. Radl S, Sundaresan S. A drag model for filtered Euler-Lagrange simulations of clustered gas-particle suspensions. Chem Eng Sci. 2014; 117:416-425. doi:10.1016/j.ces.2014.07.011.

51. Kloss C, Goniva C, Hager A, Amberger S, Pirker S. Models, algorithms and validation for opensource DEM and CFD-DEM. Prog Comput Fluid Dyn. 2012;12:140-152. doi:10.1504/ PCFD.2012.047457.

\section{Appendix A: Analytical Solution for a Two-Particle System}

The mass balance equations detailed in the manuscript can be normalized and written for the simplest case of a two-particle collision:

$$
\begin{gathered}
\frac{d L_{p 1}^{+}}{d t^{+}}=-a_{i}\left(L_{p 1}^{+} \phi_{m 1}-\frac{V_{b}^{+}}{2}\right) \\
\frac{d L_{p 2}^{+}}{d t^{+}}=-a_{i}\left(L_{p 2}^{+} \phi_{m 2}-\frac{V_{b}^{+}}{2}\right) \\
\frac{d V_{b}^{+}}{d t^{+}}=-\left(\frac{d L_{p 1}^{+}}{d t^{+}}+\frac{d L_{p 2}^{+}}{d t^{+}}\right)
\end{gathered}
$$

The initial conditions are:

$$
\left.V_{b}^{+}\right|_{t=0}=V_{b, 0}^{+},\left.L_{p 1}^{+}\right|_{t=0}=L_{p 1,0}^{+},\left.L_{p 2}^{+}\right|_{t=0}=L_{p 2,0}^{+}
$$

Exploiting the total mass balance, that is, Eq. A3, assuming that $a_{i}$ is a constant for the pair of particles, and taking the time derivatives of the above equations, we arrive at:

$$
\begin{aligned}
& \frac{d L_{p 1}^{+\prime}}{d t^{+}}=-a_{i}\left(\left(\phi_{m 1}+\frac{1}{2}\right) L_{p 1}^{+\prime}+\frac{1}{2} L_{p 2}^{+\prime}\right) \\
& \frac{d L_{p 2}^{+\prime}}{d t^{+}}=-a_{i}\left(\left(\phi_{m 2}+\frac{1}{2}\right) L_{p 2}^{+\prime}+\frac{1}{2} L_{p 1}^{+\prime}\right)
\end{aligned}
$$

where $L_{p 1}^{+\prime}=\frac{d L_{p 1}^{+}}{d t^{+}}, L_{p 2}^{+\prime}=\frac{d L_{p 2}^{+}}{d t^{+}}$. By rearranging Eq. A5, we now

$$
L_{p 2}^{+\prime}=-2\left(\frac{1}{a_{i}} \frac{d L_{p 1}^{+\prime}}{d t^{+}}+\left(\phi_{m 1}+\frac{1}{2}\right) L_{p 1}^{+\prime}\right)
$$

We then substitute Eq. A7 into Eq. A6, to arrive at the fol- 1383 lowing second-order constant coefficient homogeneous linear 1384 differential equation in for $L_{p 1}^{+\prime}$,

$$
\begin{aligned}
& \frac{2}{a_{i}} \frac{d^{2} L_{p 1}^{+\prime}}{d t^{+2}}+2\left(\phi_{m 1}+\phi_{m 2}+1\right) \frac{d L_{p 1}^{+\prime}}{d t^{+}} \\
& +a_{i}\left(2 \phi_{m 1} \phi_{m 2}+\phi_{m 1}+\phi_{m 2}\right) L_{p 1}^{+\prime}=0
\end{aligned}
$$

Using the Ansatz

$$
L_{p 1}^{+\prime} \equiv e^{r t^{+}}
$$

We obtain the characteristic equation

$$
\begin{aligned}
& \frac{2}{a_{i}} r^{2} e^{r t^{+}}+2\left(\phi_{m 1}+\phi_{m 2}+1\right) r e^{r t^{+}} \\
+ & a_{i}\left(2 \phi_{m 1} \phi_{m 2}+\phi_{m 1}+\phi_{m 2}\right) e^{r t^{+}}=0
\end{aligned}
$$

which has the real-numbered roots:

$$
\begin{array}{r}
r_{1}=\frac{-a_{i}\left(\phi_{m 1}+\phi_{m 2}+1+\sqrt{\left(\phi_{m 1}-\phi_{m 2}\right)^{2}+1}\right)}{2} \\
r_{2}=\frac{-a_{i}\left(\phi_{m 1}+\phi_{m 2}+1-\sqrt{\left(\phi_{m 1}-\phi_{m 2}\right)^{2}+1}\right)}{2}
\end{array}
$$

Hence, the general solution for Eq. A8 is given by

$$
\frac{L_{p 1}^{+}}{d t^{+}}=C_{1} e^{r_{1} t^{+}}+C_{2} e^{r_{2} t^{+}}
$$

and

$$
\begin{aligned}
\frac{L_{p 2}^{+}}{d t^{+}} & =-2\left(\frac{1}{a_{i}}\left(C_{1} r_{1} e^{r_{1} t^{+}}+C_{2} r_{2} e^{r_{2} t^{+}}\right)\right. \\
& \left.+\left(\phi_{m 1}+\frac{1}{2}\right)\left(C_{1} e^{r_{1} t^{+}}+C_{2} e^{r_{2} t^{+}}\right)\right)
\end{aligned}
$$

$\frac{d V_{b}^{+}}{d t^{+}}=\left(\frac{2 r_{1}}{a_{i}}+2 \phi_{m 1}\right) C_{1} e^{r_{1} t^{+}}+\left(\frac{2 r_{2}}{a_{i}}+2 \phi_{m 1}\right) C_{2} e^{r_{2} t^{+}}$

Using the initial conditions, that is, $\left.V_{b}^{+}\right|_{t=0}=V_{b, 0}^{+}, 1391$ $\left.L_{p 1}^{+}\right|_{t=0}=L_{p 1,0}^{+}$, and $\left.L_{p 2}^{+}\right|_{t=0}=L_{p 2,0}^{+}$, and after rearrangement we 1392 arrive at:

$$
\begin{aligned}
C_{1}= & \frac{-a_{i}^{2} \phi_{m 2} L_{p 2,0}^{+}-\left(2 a_{i} r_{2} \phi_{m 1}+2 a_{i}^{2} \phi_{m 1}^{2}+a^{2} \phi_{m 1}\right) L_{p 1,0}^{+}}{2\left(r_{2}-r_{1}\right)} \\
& +\frac{\left(a_{i}^{2} \phi_{m 1}+a_{i}^{2}+a_{i} r_{2}\right) V_{b, 0}^{+}}{2\left(r_{2}-r_{1}\right)}
\end{aligned}
$$
obtain an expression of $L_{p 2}^{+}$in terms of $L_{p 1}^{+1}$

$$
C_{2}=\frac{a_{i}^{2} \phi_{m 2} L_{p 2,0}^{+}+\left(2 a_{i} r_{1} \phi_{m 1}+a_{i}^{2} \phi_{m 1}+2 a_{i}^{2} \phi_{m 1}^{2}\right) L_{p 1,0}^{+}-\left(a_{i} r+a_{i}^{2} \phi_{m 1}+a_{i}^{2}\right) V_{b, 0}^{+}}{2\left(r_{2}-r_{1}\right)}
$$




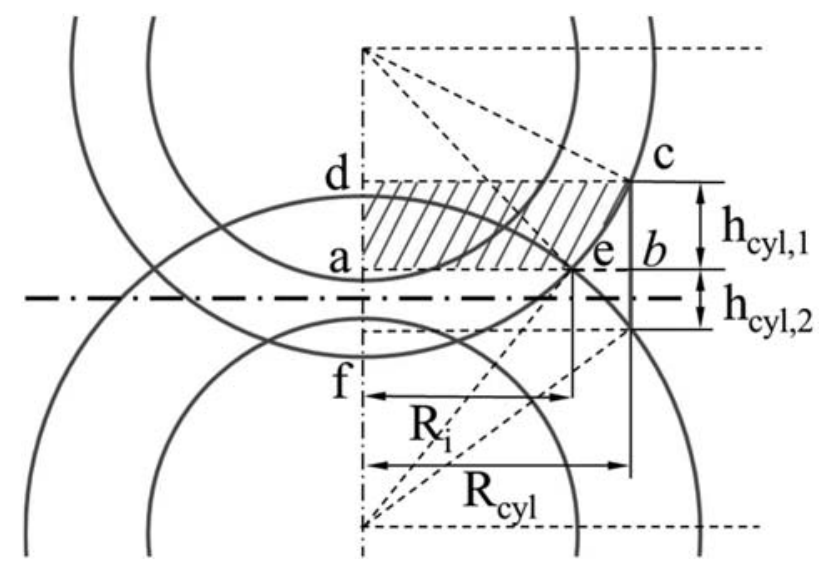

Figure B1. Sketch illustrating the unknown variables in the calculation of the geometrical bridge volume type II.

[Color figure can be viewed in the online issue, which is available at wileyonlinelibrary.com.]

by integration of Eq. A15, as well as using the initial condi-

$$
V_{b}^{+}=V_{b, 0}^{+}+C_{1} \frac{\frac{2 r_{1}}{a_{i}}+2 \phi_{m 1}}{r_{1}}\left(e^{r_{1} t^{+}}-1\right)+C_{2} \frac{\frac{2 r_{2}}{a_{i}}+2 \phi_{m 1}}{r_{2}}\left(e^{r_{2} t^{+}}-1\right)
$$

Similarly, we obtain the following equations for the dimensionless liquid content on particle 1 and particle 2:

$$
\begin{aligned}
L_{p 1}^{+}= & L_{p 1,0}^{+}+\frac{C_{1}}{r_{1}}\left(e^{r_{1} t^{+}}-1\right)+\frac{C_{2}}{r_{2}}\left(e^{r_{2} t^{+}}-1\right) \\
L_{p 2}^{+} & =L_{p 2,0}^{+}-C_{1} \frac{\frac{2 r_{1}}{a_{i}}+2 \phi_{m 1}+1}{r_{1}}\left(e^{r_{1} t^{+}}-1\right) \\
& -C_{2} \frac{\frac{2 r_{2}}{a_{i}}+2 \phi_{m 1}+1}{r_{2}}\left(e^{r_{2} t^{+}}-1\right)
\end{aligned}
$$

\section{Appendix B: Details on the Geometrical Bridge Volume Type II}

The key to calculate the volume of liquid bridges of type II is the calculation of the radius $R_{\text {cyl }}$ of the compensation cylinder region. The known parameters, as shown in Figure 1 (panel a), are the particle radius $R$, the initial film heights $h_{1}$ and $h_{2}$, and the half separation between particles $S$. Once two particles have been fixed in space, the liquid in the overlap regions of the liquid films (i.e., the red and green shaded regions in Figure 1, panel b) is displaced and flows into a ring-shaped region (i.e., red solid area in Figure 1, panel b). One can compute the volume of the ring-shaped region as follows:

First, the overlap liquid volume that is contributed by particle 1 (i.e., the green shaded area in Figure 1, panel b) and particle 2 (i.e., the red shaded area in Figure 1, panel b) can be computed from:

$$
\begin{gathered}
V_{\text {overLap }}=\frac{1}{24} \frac{1}{R+S}\left(\pi \left(12 h_{1}^{2} R^{2}-24 h_{1}^{2} S^{2}+4 R h_{1}^{3}+16 h_{1}^{3} S-3 h_{1}^{4}-48 R^{2} h_{1} S\right.\right. \\
-48 R h_{1} S^{2}+96 R^{2} S^{2}+128 R S^{3}+32 S^{4}+12 R^{2} h_{2}^{2}-24 h_{2}^{2} S^{2}+4 R h_{2}^{3} \\
\left.\left.+16 h_{2}^{3} S-3 h_{2}^{4}-48 R^{2} h_{2} S-48 R h_{2} S^{2}\right)\right)
\end{gathered}
$$

Second, the intersection radius $R_{i}$ can be calculated using Eq. B2 as illustrated in Figure B1B1.

$$
\sqrt{\left(R+h_{1}\right)^{2}-R_{i}^{2}}+\sqrt{\left(R+h_{2}\right)^{2}-R_{i}^{2}}=2(R+S)
$$

Third, the ring-shaped region is divided into two sub-regions, 1417 which have the heights $h_{\mathrm{cyl}, 1}$ and $h_{\mathrm{cyl}, 2}$ as sketched in Figure B1. 1418 The ring-shaped sub-region 1 has the volume $V_{\text {cyl,1 }}=V_{\text {abcd }}-1419$ $V_{\text {aecd, }}$ where $V_{\text {aecd }}=V_{\text {dcf }}-V_{\text {cap,aef. }}$ The cap height is 1420 $h_{\mathrm{af}}=R+h_{1}-\sqrt{\left(R+h_{1}\right)^{2}-R_{i}^{2}}$, and thus the cap volume is 1421 $V_{\text {cap,aef }}=\frac{\pi h_{\mathrm{aff}}}{6}\left(3 R_{i}^{2}+h_{\mathrm{af}}^{2}\right)$. The liquid volume in sub-region 2 , that 1422 is, $V_{\text {cyl,2 }}$ can be computed in the same fashion.

Finally, we sum up the two sub-regions, and use the known 1424 volume of the displaced liquid, that is, $V_{\text {overLap }}$, to arrive at the 1425 following expression involving the unknowns $R_{\text {cyl }}, h_{1}, h_{2}, S, 1426$ and $R$ :

$$
V_{\text {overLap }}=V_{\mathrm{cyl}}=V_{\mathrm{cyl}, 1}+V_{\mathrm{cyl}, 2}=f\left(R_{\mathrm{cyl}}, h_{1}, h_{2}, S, R\right)
$$

This expression cannot be solve directly to obtain $R_{\text {cyl }}$, how- 1428 ever, one can solve it numerically using, for example, a Newton 1429 algorithm. Once $R_{\text {cyl, }}$ is known, we can calculate the geometrical 1430 bridge volume based on the contribution from each particle $i 1431$ and as illustrated in shown in Figure 4 (panel b):

$$
\beta_{\mathrm{cyl}, i}=\arcsin \left(\frac{R_{\mathrm{cyl}}}{R+h_{i}}\right)
$$

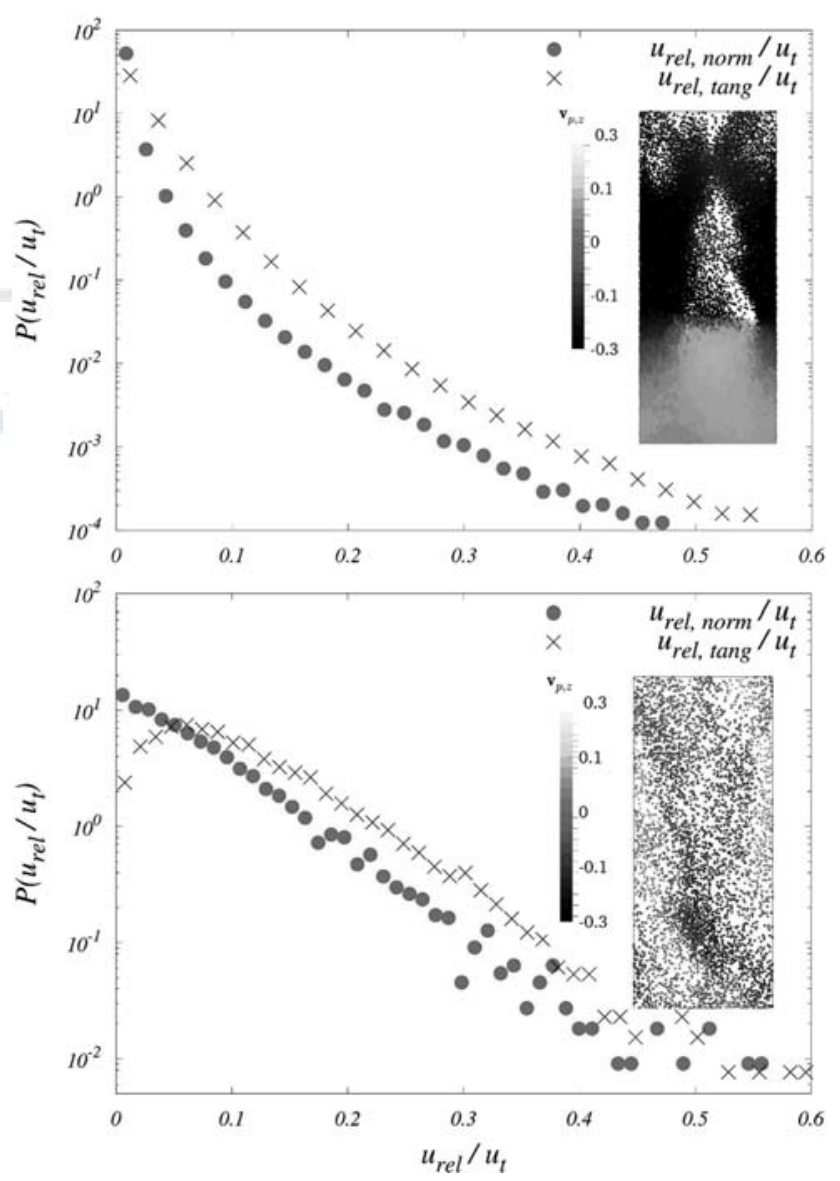

Figure C1. Distribution of the relative particle velocity at impact in a dilute (bottom panel; $\phi_{p}=0.05$ ) and dense (top panel; $\phi_{p}=0.30$ ) cloud of freely sedimenting particles (the inserts illustrate individual-particle velocities in the vertical direction).

[Color figure can be viewed in the online issue, which is available at wileyonlinelibrary.com.] 


$$
\begin{gathered}
h_{\mathrm{cyl}, i}=(R+S)-\left(R+h_{i}\right) \cos \beta_{\mathrm{cyl}, i} \\
V_{\mathrm{cap}, i}=\frac{\pi R^{3}}{3}\left(1-\cos \beta_{\mathrm{cyl}, i}\right)^{2}\left(2+\cos \beta_{\mathrm{cyl}, i}\right) \\
V_{\mathrm{b}, i, \mathrm{I}}=\pi R_{\mathrm{cyl}}^{2} h_{\mathrm{cyl}, i}+\frac{1}{3} \pi h_{i} \cos \beta_{\mathrm{cyl}, i} \sin ^{2} \beta_{\mathrm{cyl}, i} \\
\left(R^{2}+\left(R+h_{i}\right)^{2}+R\left(R+h_{i}\right)\right) \\
V_{\mathrm{b}, \mathrm{g}, \mathrm{II}, i}=V_{\mathrm{b}, i, \mathrm{I}}-V_{\mathrm{cap}, i}
\end{gathered}
$$

\section{3 \\ Appendix C: Relative Particle Velocity at Impact in a Sedimenting Suspension}

We summarize statistics of the relative particle speed at impact in a fully periodic domain using soft-sphere Euler-
Lagrange simulations using the code $\mathrm{CFDEM}^{\circledR}$ (Kloss et al. ${ }^{51}$ ). 1438 Particles were allow to sediment under the action of gravity, 1439 while their weight (and that of the surrounding gas) was bal- 1440 anced by a pressure gradient, similar to our previous work (see, 1441 Radl and Sundaresan ${ }^{50}$ ). Also numerical parameters and drag 1442 models were identical to this previous work (a dimensionless 1443 grid resolution of $\Delta x /(2 R)=3$, as a domain size of $53 \times 53 \times 1444$ $213 d_{p}$ was used). The statistics reported below were collected 1445 by sampling impact velocities over a sufficiently long time, that 1446 is, 40 times the particle relaxation time $t_{\text {relax }}=u_{t}^{2} / g$. Note that 1447 the particles were initialized homogeneously distributed in the 1448 computational domain, and that a statistical steady state was 1449 reached after ca. $5 t_{\text {relax }}$. 
AQ1: Please provide page range for Refs. 6, 24, and 29.

AQ2: Please provide complete publication details for Ref. 30.

AQ3: Please provide location details for Ref. 44.

AQ4: Please confirm that given names (red) and surnames/family names (green) have been identified correctly.

Ref. 6. Dodds S, Carvalho M, Kumar S. Stretching liquid bridges with moving contact lines: The role of inertia. Phys Fluids. $2011 ; 23(9): 092101$. doi:10.1063/1.3623427.

Ref 24. Donahue CM, Hrenya CM, Davis RH. Stokes's cradle: Newton's cradle with liquid coating. Phys Rev Lett. 2010;105(3):034501. doi: 10.1103/PhysRevLett.105.034501.

Ref 29. Kantak AA, Hrenya MC, Davis HR. Initial rates of aggregation for dilute, granular flows of wet particles. Phys Fluids. 2009;21(2):023301. doi: 10.1063/1.3070830.

Ref. 30. Yang F. Interaction Law for a Collision Between Two Solid Particles in a Viscous Liquid. (Ph D) Thesis Calif Inst Technol. 2006. doi: etd-05262006-120244.

Ref. 44. Easo LA, Kumar R, Ren R, Carl R. Numerical Study of the Formation of a Liquid Bridge Between Two Spheres with Uniform Film Thickness. In: 2014 AICHE Annual Meeting, November 18. Atlanta; 2014. 